\title{
PSTEP: project for solar-terrestrial environment prediction
}

\author{
Kanya Kusano ${ }^{*} \mathbb{0}$, Kiyoshi Ichimoto ${ }^{2}$, Mamoru Ishii ${ }^{3}$, Yoshizumi Miyoshi ${ }^{4}$, Shigeo Yoden ${ }^{5}$, Hideharu Akiyoshi ${ }^{6}$, \\ Ayumi Asai ${ }^{7}$, Yusuke Ebihara ${ }^{8}$, Hitoshi Fujiwara ${ }^{9}$, Tada-Nori Goto ${ }^{10}$, Yoichiro Hanaoka ${ }^{11}$, Hisashi Hayakawa ${ }^{4}$, \\ Keisuke Hosokawa ${ }^{12}$, Hideyuki Hotta ${ }^{13}$, Kornyanat Hozumi ${ }^{3}$, Shinsuke Imada', Kazumasa Iwai ${ }^{4}$, \\ Toshihiko lyemori ${ }^{14}$, Hidekatsu Jin ${ }^{3}$, Ryuho Kataoka ${ }^{15}$, Yuto Katoh ${ }^{16}$, Takashi Kikuchi ${ }^{4}$, Yûki Kubo ${ }^{17}$, \\ Satoshi Kurita ${ }^{8}$, Haruhisa Matsumoto ${ }^{18}$, Takefumi Mitani ${ }^{19}$, Hiroko Miyahara ${ }^{20}$, Yasunobu Miyoshi ${ }^{21}$, \\ Tsutomu Nagatsuma ${ }^{22}$, Aoi Nakamizo ${ }^{3}$, Satoko Nakamura ${ }^{4}$, Hiroyuki Nakata ${ }^{23}$, Naoto Nishizuka ${ }^{3}$, Yuichi Otsuka ${ }^{4}$, \\ Shinji Saito ${ }^{3}$, Susumu Saito ${ }^{24}$, Takashi Sakurai ${ }^{11}$, Tatsuhiko Sato ${ }^{25}$, Toshifumi Shimizu ${ }^{19}$, Hiroyuki Shinagawa ${ }^{3}$, \\ Kazuo Shiokawa ${ }^{4}$, Daikou Shiota ${ }^{3}$, Takeshi Takashima ${ }^{19}$, Chihiro Tao ${ }^{3}$, Shin Toriumi ${ }^{19}$, Satoru Ueno ${ }^{26}$, \\ Kyoko Watanabe ${ }^{27}$, Shinichi Watari ${ }^{3}$, Seiji Yashiro ${ }^{28}$, Kohei Yoshida ${ }^{29}$ and Akimasa Yoshikawa ${ }^{30}$
}

\begin{abstract}
Although solar activity may significantly impact the global environment and socioeconomic systems, the mechanisms for solar eruptions and the subsequent processes have not yet been fully understood. Thus, modern society supported by advanced information systems is at risk from severe space weather disturbances. Project for solar-terrestrial environment prediction (PSTEP) was launched to improve this situation through synergy between basic science research and operational forecast. The PSTEP is a nationwide research collaboration in Japan and was conducted from April 2015 to March 2020, supported by a Grant-in-Aid for Scientific Research on Innovative Areas from the Ministry of Education, Culture, Sports, Science and Technology of Japan. By this project, we sought to answer the fundamental questions concerning the solar-terrestrial environment and aimed to build a next-generation space weather forecast system to prepare for severe space weather disasters. The PSTEP consists of four research groups and proposal-based research units. It has made a significant progress in space weather research and operational forecasts, publishing over 500 refereed journal papers and organizing four international symposiums, various workshops and seminars, and summer school for graduate students at Rikubetsu in 2017. This paper is a summary report of the PSTEP and describes the major research achievements it produced.
\end{abstract}

Keywords: Solar physics, Solar-terrestrial environment, Space weather, Earth environmental system

\section{Introduction}

The solar-terrestrial environment, including the Sun, the Earth, and the interplanetary space, is the place of modern civilization's activity. As a result of the progress of space exploration and the rapid growth of information

\footnotetext{
${ }^{*}$ Correspondence: kusano@nagoya-u.jp

${ }^{1}$ Institute for Space-Earth Environmental Research (ISEE), Nagoya

University, Nagoya 464-8601, Japan

Full list of author information is available at the end of the article
}

society, our socioeconomic system supported by highly developed infrastructures, e.g., communication systems, electric power grid, aviation network, and artificial satellites, may become more vulnerable to space weather and space climate variabilities (see Fig. 1).

Recent studies suggested that an extreme space weather event equivalent to the Carrington Event, which occurred on September 1, 1859, is likely to cause significant social impacts (National Research Council 2008). Furthermore, the cosmogenic isotope analysis recorded in tree rings 


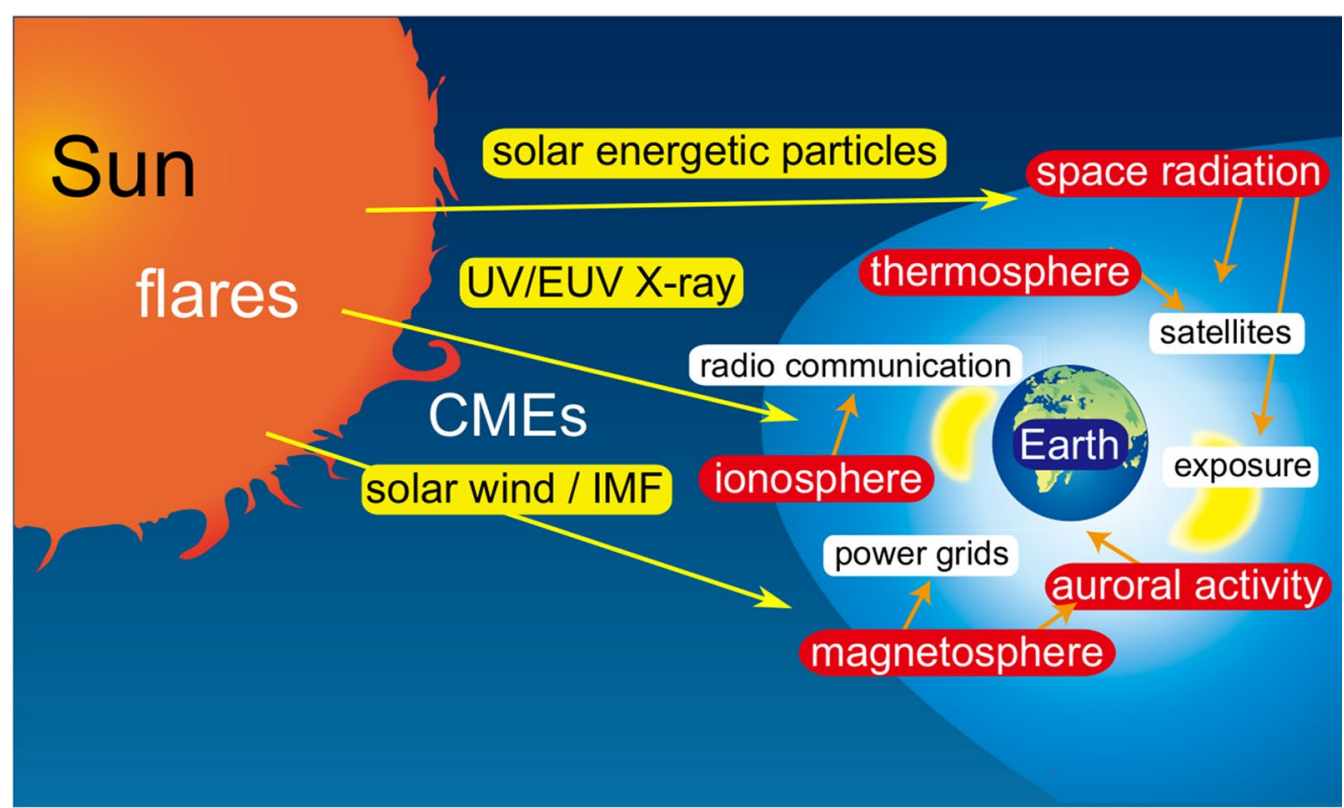

Fig. 1 The illustration of solar-terrestrial environment. Solar flares and coronal mass ejections (CMEs) may impact our infrastructures through the disturbances in magnetosphere, ionosphere and thermosphere

and ice-core indicates the probability that the Sun may have caused massive explosions of more than ten times the Carrington Event (Miyake et al. 2012). Therefore, space weather disturbance is a potential risk to modern society.

Also, the mechanisms by which long-term solar activity variations affect the Earth's weather and climate are still unclear. Thus, a significant uncertainty remains in the assessment of solar impacts in climate change projections. These facts suggest that a planetary-scale environmental change caused by the Sun may be a dangerous phenomenon and there is an urgent need to establish a scientific basis for accurate prediction of space weather and space climate. However, we have not yet gained sufficient predictive capability of the solar-terrestrial environment. There are two possible major reasons that hamper the progress in our prediction, which are as follows:

First, there is a lack of full-fledged interdisciplinary research. Dynamics in the solar-terrestrial environment are complex phenomena studied in separate disciplines, and there has been an insufficient attempt to grasp the whole picture of the events.

Second, there is the gap between scientific research and operational forecasts. Government agencies in various countries are monitoring the solar-terrestrial environment and operating the prediction of changes in it. However, current space weather forecasting remains at the stage of relying on limited past experiences. We have not yet reached the stage of precise and robust predictions based on physics. This situation has been described as a "valley of death" between cutting-edge space science and space weather forecasting. While scientific research has not yet reached the level of application in operational forecasting, many scientists are not interested in the ad hoc prediction that relies only on heuristic methods. It has created a mutually negative perception between the scientific understanding and the forecasting efforts of the solar-terrestrial environmental dynamics.

Therefore, both scientifically and practically, it is an important and urgent issue to develop a synergy between scientific research and operational forecasts, collaborating among the various disciplines related to space weather and space climate. Project for Solar-Terrestrial Environment Prediction (PSTEP) was launched in 2015 to obtain the synergy between basic sciences and operational forecasts. The PSTEP is a nationwide research collaboration for 5 years supported by a Grant-in-Aid for Scientific Research on Innovative Areas from the Ministry of Education, Culture, Sports, Science and Technology of Japan. This project sought to answer some of the fundamental questions concerning the solar-terrestrial environmental and aimed to contribute to building a next-generation space weather forecast system to prepare for severe space weather disasters.

The goal of this project was to achieve the following two objectives: 
1. By integrating the latest observations and simulations, we would build a new scheme to predict phenomena based on a physical model, and through a quantitative verification of the predictions, we would improve our scientific understanding and finally solve critical scientific problems such as the onset mechanism of solar flares, the mechanism of ionospheric and magnetospheric disturbances, and the variability of the solar cycle and their influences on meteorology and climate.

2. Through cross-disciplinary research, we would investigate the impact of the solar-terrestrial environment dynamics on the social systems and provide feedbacks from the quantitative validation of the forecast to improve our scientific understanding of the solar-terrestrial environment. To this end, we would build an interactive system that can allow us to make the best use of space weather forecasting as a social infrastructure. For instance, we would develop space weather benchmarks for severe space weather disasters based on state-of-the-art scientific research.

The PSTEP consisted of the following four research groups (A01-A04, Fig. 2): the goal of the Group for Space Weather Forecast System (A01) was to implement the physics-based model developed by the other groups A02-A04 and build the system to provide a forecast result to the society that needs them. Also, this group would make a space weather hazard map of Japan. Furthermore, this group would develop a next-generation space weather forecast model which can realize more

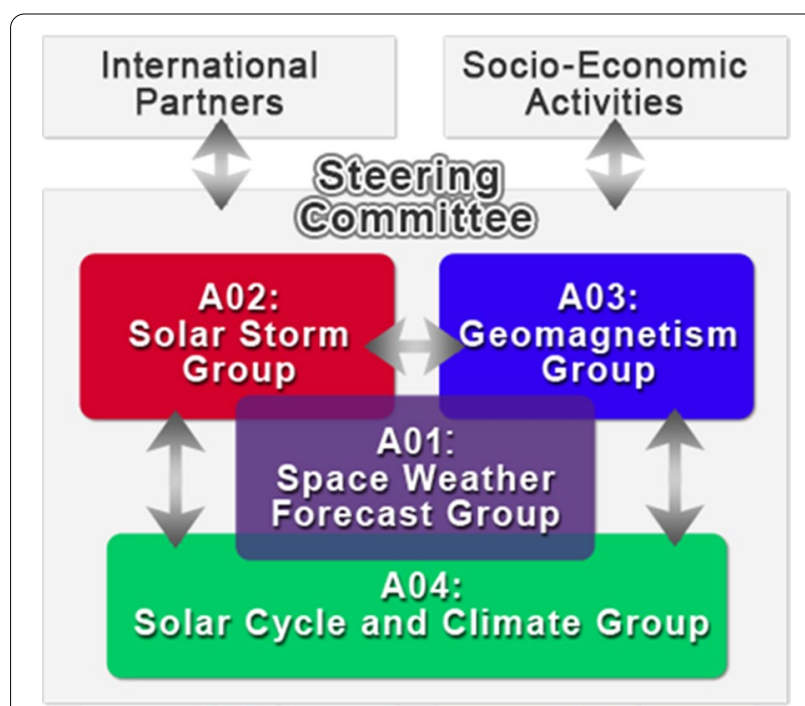

Fig. 2 PSTEP is organized by four research groups (A01-A04). With collaborations with international partners and socioeconomic activities precise predictions based on physics-based models in real-time.

The goal of Solar Storm Group (A02) was to develop the prediction of solar flares and solar eruptions by combining the physics-based models and the data observed by the Hinode satellite and other space-borne and ground-based instruments. By elucidating the triggering mechanism, we would develop a more accurate scheme for predicting large solar flares. The fundamental goal was to create a methodology for probabilistic prediction within a few hours of the onset of a large flare. Furthermore, this group would develop an appropriate assimilation method of observations and numerical models to predict the impact of a coronal mass ejection (CME) before it arrives the Earth.

The goal of Space Dynamics Group (A03) was to improve the accuracy of the predictions of the following three phenomena in the Earth's magnetosphere and ionosphere: (1) thermospheric density and ionospheric electron density variations caused by solar flares several hours in advance and the probability of occurrence and arrival of ionospheric mesoscale disturbance phenomena such as plasma bubbles; (2) geomagnetically induced currents (GICs) in Japan, and (3) variation of cosmic radiation, in particular the variation of high-energy particles in the radiation belts in the Earth's magnetosphere.

The Group for Solar Cycle Activity and Impact on Climate (A04) aimed (1) to predict the activity of the next solar cycle (i.e., Cycle 25) and (2) to improve the understanding of the mechanisms of solar impact on climate. This group would also elucidate the mechanisms of longterm solar activity variability on a multihundred-year scale and incorporate solar effects and simulate longterm climate changes caused by solar activity variation using the Earth system model.

The PSTEP was conducted from April 2015 to March 2020 and produced many scientific results, including more than 500 refereed journal papers and many new models for predicting solar-terrestrial environmental processes (see Table 1). This paper is the summary report of the PSTEP research activity, and the rest of the paper is organized as follows: in Sects. 2, 3, 4 and 5, the main results of the PSTEP are, respectively, shown for each research group. In Sect. 6, finally, the contribution of the PSTEP to the understanding and prediction of space weather and space climate is summarized, and future issues are discussed.

\section{Main results of A01 group: space weather forecast system}

Our basic objective of A01 group was to establish a system to provide detailed space weather information to the public by interconnecting the physical models 
Table 1 The models developed by PSTEP for solar-terrestrial environment prediction

\begin{tabular}{|c|c|c|c|}
\hline Model name & Description & URL & References \\
\hline $\mathrm{R} 2 \mathrm{D} 2$ & $\begin{array}{l}\text { Radiation and reduced speed of sound } \\
\text { technique for deep dynamics model of solar } \\
\text { convection }\end{array}$ & https://hottahd.github.io/R2D2-manual/\# & Hotta et al. (2019) \\
\hline k-scheme & $\begin{array}{l}\text { A physics-based model to predict imminent } \\
\text { large solar flares }\end{array}$ & $\begin{array}{l}\text { https://nagoya.repo.nii.ac.jp/records/ } \\
\text { 30123\#.YJDXm7X7SF4 }\end{array}$ & Kusano et al. (2020) \\
\hline Deep Flare Net (DeFN) & $\begin{array}{l}\text { A solar flare prediction model using deep } \\
\text { neural networks }\end{array}$ & https://defn.nict.go.jp/index_eng.html & Nishizuka et al. (2018) \\
\hline Solar flare emission model & $\begin{array}{l}\text { A model for reproducing } X \text {-rays and EUV emis- } \\
\text { sions from solar flares }\end{array}$ & & $\begin{array}{l}\text { Nishimoto et al. (2020) } \\
\text { Nishimoto et al. (2021) } \\
\text { Kawai et al. (2020) }\end{array}$ \\
\hline NonFFF model & $\begin{array}{l}\text { A realistic non-force-free field modeling of } \\
\text { three-dimensional magnetic field in the solar } \\
\text { corona }\end{array}$ & & Miyoshi et al. (2020) \\
\hline SUSANOO & $\begin{array}{l}\text { A three-dimensional MHD simulation of } \\
\text { propagation of solar wind and coronal mass } \\
\text { ejections in the inner heliosphere }(r<2 \mathrm{AU}) \\
\text { on the basis of photospheric magnetic field } \\
\text { observations. The modeled time profile of } \\
\text { solar wind at the Earth's position is utilized } \\
\text { for a prediction of high-energy electron flux } \\
\text { at the geosynchronous orbit }\end{array}$ & http://cidas.isee.nagoya-u.ac.jp/susanoo/ & $\begin{array}{l}\text { Shiota and Kataoka (2016) } \\
\text { Shiota (2020) }\end{array}$ \\
\hline Solar cycle prediction & $\begin{array}{l}\text { A solar cycle prediction model using a surface } \\
\text { flux transport model }\end{array}$ & & lijima et al. (2017) \\
\hline Magnetosphere global model & $\begin{array}{l}\text { A global magnetohydrodynamics (MHD) } \\
\text { model of geo-magnetosphere and iono- } \\
\text { sphere system }\end{array}$ & & Tanaka (2015) \\
\hline WASAVIES & $\begin{array}{l}\text { A physics-based model for SEP dose estima- } \\
\text { tion anywhere in the atmosphere and } \\
\text { designated as a warning system for aviation } \\
\text { exposure to solar energetic particles }\end{array}$ & https://wasavies.nict.go.jp/ & $\begin{array}{l}\text { Sato et al. (2018a) } \\
\text { Kataoka et al. (2018) }\end{array}$ \\
\hline SECURES & $\begin{array}{l}\text { Space Environment Customized Risk Estima- } \\
\text { tion for Satellites }\end{array}$ & & Nagatsuma et al. (2021) \\
\hline MUSCAT & Multi-Utility Spacecraft Charging Analysis Tool & & $\begin{array}{l}\text { Hosoda et al. (2008) } \\
\text { Muranaka et al. (2008 }\end{array}$ \\
\hline GAIA & $\begin{array}{l}\text { A whole atmosphere GCM which solves physi- } \\
\text { cal and chemical processes from the ground } \\
\text { to upper atmosphere under interaction with } \\
\text { the ionosphere }\end{array}$ & https://gaia-web.nict.go.jp/index_e.html & $\begin{array}{l}\text { Jin et al. (2012) } \\
\text { Tao et al. (2020) }\end{array}$ \\
\hline Plasma bubble model & $\begin{array}{l}\text { This model is able to reproduce nonlinear } \\
\text { growth of equatorial plasma bubbles by } \\
\text { solving the electrodynamics of equatorial } \\
\text { ionosphere with very high spatial resolution }\end{array}$ & & Yokoyama et al. (2019) \\
\hline HF-START & $\begin{array}{l}\text { High Frequency Simulator Targeting for All } \\
\text { Users'Regional Telecommunications }\end{array}$ & https://hfstart.nict.go.jp/ & Hozumi et al. (2019) \\
\hline GIC model & $\begin{array}{l}\text { Time Domain Simulation of Geomagnetically } \\
\text { Induced Current (GIC) }\end{array}$ & & Nakamura et al. (2018) \\
\hline Space weather benchmarks & $\begin{array}{l}\text { The plausible space weather disasters in Japan } \\
\text { with scale and frequency are described and } \\
\text { estimated from past studies }\end{array}$ & $\begin{array}{l}\text { https://www2.nict.go.jp/spe/benchmark/ } \\
\text { (in Japanese) }\end{array}$ & Ishii et al. (2021) \\
\hline
\end{tabular}

developed by groups A02-A04 with the space weather forecast information required by the public. We have developed different models for space weather prediction and also published space weather hazard maps of Japan as described in the following subsection. They would contribute to the establishment of a next-generation space weather forecast system that can provide precise predictions based on physical models in real-time.

\section{Discussion of required space weather information with users}

We established "Space Weather users committee" for discussing the needs-seeds matching in space weather 
services and picked up the requirements from the space weather information users and the scientific products derived from scientists. From the viewpoint of operational demands, users request long-term space weather prediction, for example, solar flare occurrence for about 27 days, GIC peak time for 2 days, and ionospheric disturbances for $30 \mathrm{~h}$. They are beyond the predictive timescale in our current operational forecast. Users also wish to know more accurate information than the currently possible prediction.

The gaps exist not only between the needs and the seeds of space weather forecast, but also between researchers and operators of space weather forecast. To resolve the problems, we have developed the schemes in PSTEP. Some simulation and model codes were improved in the PSTEP, e.g., Ground-to-Topside Model of Atmosphere and Ionosphere for Aeronomy (GAIA) real-time operation, SUSANOO-IPS, and Deep Flare Net (DeFN), which are used now in space weather forecast services in the National Institute of Information and Communications Technology (NICT). The cooperation and deep inter-communication between scientists and operators are also important to fully use the model for forecasts. We have developed new schemes for it. For example, an index called "I-scale" (Tao et al. 2020) which can be provided by the real-time GAIA model operation to evaluate ionospheric storms at NICT, Japan is better used for monitoring and forecast using GNSS observation (Nishioka et al. 2017) rather than simply showing ionospheric model parameters, i.e., plasma density. Also, we conducted the training for operators to understand the scientific framework of the models, e.g., the heliospheric model aided by the interplanetary scintillation measurement, SUSANOO-IPS. It helped to achieve operational prediction using the model simulations.

\section{Next-generation space weather forecast systems Radio propagation simulator}

We developed a radio propagation simulator named High Frequency Simulator Targeting for All Users' Regional Telecommunications (HF-START) (Hozumi et al. 2019) for estimating the influence of ionospheric disturbance in HF communication and broadcast and satellite positioning (Fig. 3). Because the ionosphere has a local characteristic, it is necessary to have a reconstructed local ionospheric data as a propagation medium in order to obtain a realistic radio propagation information. There are many radio propagation simulators available worldwide. However, most of them were developed based on statistical ionospheric data. The existing simulators, therefore, could not be well capable with a day-to-day variability and local perturbations of the ionosphere. One of the advantages of our system is to use real-time ionospheric condition obtained from global position system




(GPS) network in Japanese area named "GEONET". The GEONET was reconstructed by using 3D tomography technique in a real-time basis over Japan (Saito et al. 2017). Using a real-time 3D tomography makes it possible to realistically estimate the present condition of radio usability over Japan. In addition to the real-time observation data, we also employed GAIA (Jin et al. 2012; Tao et al. 2020) data as a propagation medium for a global user.

In parallel to the simulator development, we also assessed the risk of space weather on the HF radio propagation. Hozumi et al. (2019) pointed out that there might be a risk of a loss of aircraft en route communications due to the coexistence of sporadic E (Es) and mediumscale traveling ionospheric disturbances (MSTIDs). Japan is known to be a favorable location for both Es (Taguchi and Shibata 1961) and MSTIDs (Saito et al. 2007; Otsuka et al. 2007) to occur. There were limited quantitative reports on the simultaneous occurrence of the Es and MSTIDs. Bowman $(1960,1968)$ found 85\% simultaneous occurrence rate of the Es and travelling ionospheric disturbances (TIDs) from the 13 case studies in nighttime. Otsuka et al. (2008) reported cross-correlation coefficients between MSTIDs and critical frequency of Es and between MSTIDs and blanketing frequency of Es in summer nighttime during 2001-2005 over Japan. The cross-correlation coefficients, which are in the ranges of 0.48-0.65, show a good agreement of the Es and MSTIDs occurrences. By the way, the record of in-flight missing communication link is out of reach or is not available publicly. Therefore, the authors suggested to consider the space weather effect as one of the potential causes of the de facto missing of in-flight communication link especially in summer nighttime. Without the space weather knowledge, aeronautical users might regard such a missing communication as an instrumental failure. Unnecessary replacement of the radio frequency receiver onboard will be performed for quality assurance of the aircraft communication system. However, the pointed-out risk is not widely known and it may be just a theoretical risk that needs evaluation.

To evaluate the radio propagation time from HFSTART, we developed a digital receiver, Low-cost Amateur receiver System Employing RTL-SDR (LASER) based on RTL-SDR (https://www.rtl-sdr.com) that is a cheap open-source hardware and GNU Radio (http://gnuradio. $\mathrm{org} /$ ) that is an open-source software toolkit (Hozumi et al. 2018). The evaluation campaign was launched in late 2018 by installing the developed LASER at Wakkanai, Kokubunji, Yamagawa, and Okinawa to receive the HF radios transmitted from the Radio NIKKEI in Nagara. More details on the development and the campaign are available in the paper by Hozumi et al. (2018).
The HF-START web system has been successfully developed and is now available at https://hfstart.nict.go. jp/. On March 24, 2021, two main functions of the HFSTART service, which are (1) real-time information provision and (2) web calculation, were released. Because of its user-friendly feature, prior knowledge on space weather is not a prerequisite in order to use the service. Users can select the propagation medium from real-time observation and models. Then, users just simply input locations of transmitter and receiver, transmitting frequency and angle, date and time; and then click a run button. Soon after that, the radio propagation information on demand will be served to the users. More information on the HF-START service is available at "What is "HF-START"?" and "Readme" on the website.

\section{Estimation of individual satellite charging}

One of the major causes of satellite anomaly is charging and discharging phenomena, which are caused by disturbances in the geospace environment such as injection of substorm particles and enhancement of radiation belt particles. Substorm particles and radiation belt particles cause surface charging and internal charging, respectively. To mitigate the effect of satellite anomaly caused by charging and discharging, information of disturbances predicted by space weather forecasting needs to be known in advance. However, charging and discharging conditions differ depending on the satellite shape and materials even if the condition of space environment is the same. Therefore, it is difficult for a satellite operator to identify the operational risk of an individual satellite using only the general information of space weather. To provide tailored space weather information, we have developed SECURES (Space Environment Customized Risk Estimation for Satellites) by combining models of the space environment and those of spacecraft charging (Nagatsuma et al. 2021). SECURES consists of a surface charging part and an internal charging part. In SECURES, we focus on the risk of spacecraft charging (surface/internal) for geosynchronous satellites.

The space environment that causes surface charging is simulated by the Global Magnetohydrodynamics (MHD) model (Tanaka 2015 and references therein). To perform a more realistic simulation, we have improved the model by introducing the inclination and precession of the magnetic dipole axis (Kubota et al. 2019). With this improved model, a real-time global magnetosphere simulation was carried out. The charging condition of an individual satellite was calculated using a satellite charging analysis model such as the Spacecraft Plasma Interaction System (SPIS) (Roussel et al. 2008) or Multi-Utility Spacecraft Charging Analysis Tool (MUSCAT) (Hosoda et al. 
2008; Muranaka et al. 2008) with the simulated space environment.

The spacecraft charging analysis model requires the densities and temperatures of ions and electrons $\left(\mathrm{N}_{\mathrm{i}}\right.$, $\mathrm{T}_{\mathrm{i}}, \mathrm{N}_{\mathrm{e}}$, and $\mathrm{T}_{\mathrm{e}}$ ) as the input parameters of the model. Since MHD models cannot simulate these parameters in principle, empirical equations for estimating them using the MHD model were developed on the basis of statistical comparison between plasma parameters in GEO observed by the Magnetospheric Plasma Analyzer (MPA) onboard Los Alamos National Laboratory (LANL) satellites and those simulated by the MHD model. Furthermore, we have developed a quick estimation method of the equilibrium surface potential using precalculated results obtained from an individual geometric model, since the calculation of spacecraft charging analysis model takes hours to days for one space environment condition. We have developed two geometric models of the Van Allen Probes and the Michibiki-1 satellite as a test case.

We have confirmed that the satellite potential estimated from the surface charging part of SECURES is in relatively good agreement with the LANL satellite's observation within a certain factor (Nagatsuma et al. 2021). The results of the prototype surface charging/ ESD risk estimation system can be browsed in near real time with a web viewer.

High-energy particles that cause internal charging were predicted by radiation belt simulation using the one-dimensional Fokker-Planck equation to describe the radial diffusion (Miyoshi et al. 2004). The information on the predicted space environment was used to calculate the internal charging using MUSCAT or a simple analysis method introduced in the NASAHDBK-4002A (2017).

As an example of internal charging phenomena, the noise of the Earth Sensor Assembly (ESA) onboard the "Kodama" satellite was analyzed and confirmed to be highly correlated with the predicted value of high-energy electron flux. We tried to reproduce the internal charging on the basis of MUSCAT with a detailed model of ESA and predicted high-energy electron flux. However, we found that the calculated variation of the electrostatic potential of ESA was very small compared to the threshold potential of electrostatic discharge (ESD). This result suggests that the possibility of internal charging/ESD with other devices or cables, which are located under thin shield, needs to be considered. A detailed quantitative analysis is necessary to clarify the internal charging/ ESD of the ESA onboard Kodama, including the development of the detailed structure and material model inside the satellite.
Therefore, internal charging part of SECURES has been developed on the basis of simple analysis method introduced in the NASA-HDBK-4002A (2017). This system is capable of sending an alert of elevated risk to the user who set the thickness of the shield and the device in accordance with the ESD risk.

We have confirmed the basic functions of SECURES as prototype products. SECURES will be provided to the public in the near future. These products will be useful for satellite operators and will improve the safety of satellite operation.

\section{Estimation of human radiation dose on aviation and space station}

When a large solar flare occurs, the radiation doses due to solar energetic particle (SEP) are occasionally increased especially at flight altitudes, and they can be a potential hazard to aircrews and passenger. Thus, development of a system for nowcasting and/ or forecasting radiation doses due to SEP exposure is one of the most important challenges in space weather research. Collaboration with other research fields such as nuclear physics and radiological protection is the key issue in such development. We therefore developed a physics-based model for SEP dose estimation anywhere in the atmosphere and designated it as a warning system for aviation exposure to solar energetic particles (WASAVIES) (Sato et al. 2018a; Kataoka et al. 2018). It is an integrated system of the results of three simulations, which are as follows: (1) SEP transport simulation between the Sun and the Earth based on the focused transport equation (Kubo et al. 2015); (2) SEP transport simulation in the magnetosphere based on an empirical geomagnetic filed model, and (3) air shower simulation induced by SEP based on the PHITS code (Sato et al. 2014, 2018b).

In WASAVIES, the count rates of several neutron monitors at the ground level, as well as the proton fluxes measured by the GOES satellite, are continuously downloaded at intervals of $5 \mathrm{~min}$ and used for checking the occurrence of ground level enhancement (GLE). When a GLE event is detected, the system automatically determines the model parameters to characterize the profiles of each GLE event and nowcasts the radiation dose rates all over the world. The performance of WASAVIES is examined by analyzing the four major GLE events of the twenty-first century. Figure 4 shows the worldwide dose rate map at $12 \mathrm{~km}$ during GLE 69 (7:00 UT, January 20, 2005) drawn using WASAVIES. A web interface of WASAVIES was also developed and opened via public server of NICT (https://wasavies. nict.go.jp/) since November 7, 2019. The radiation dose 


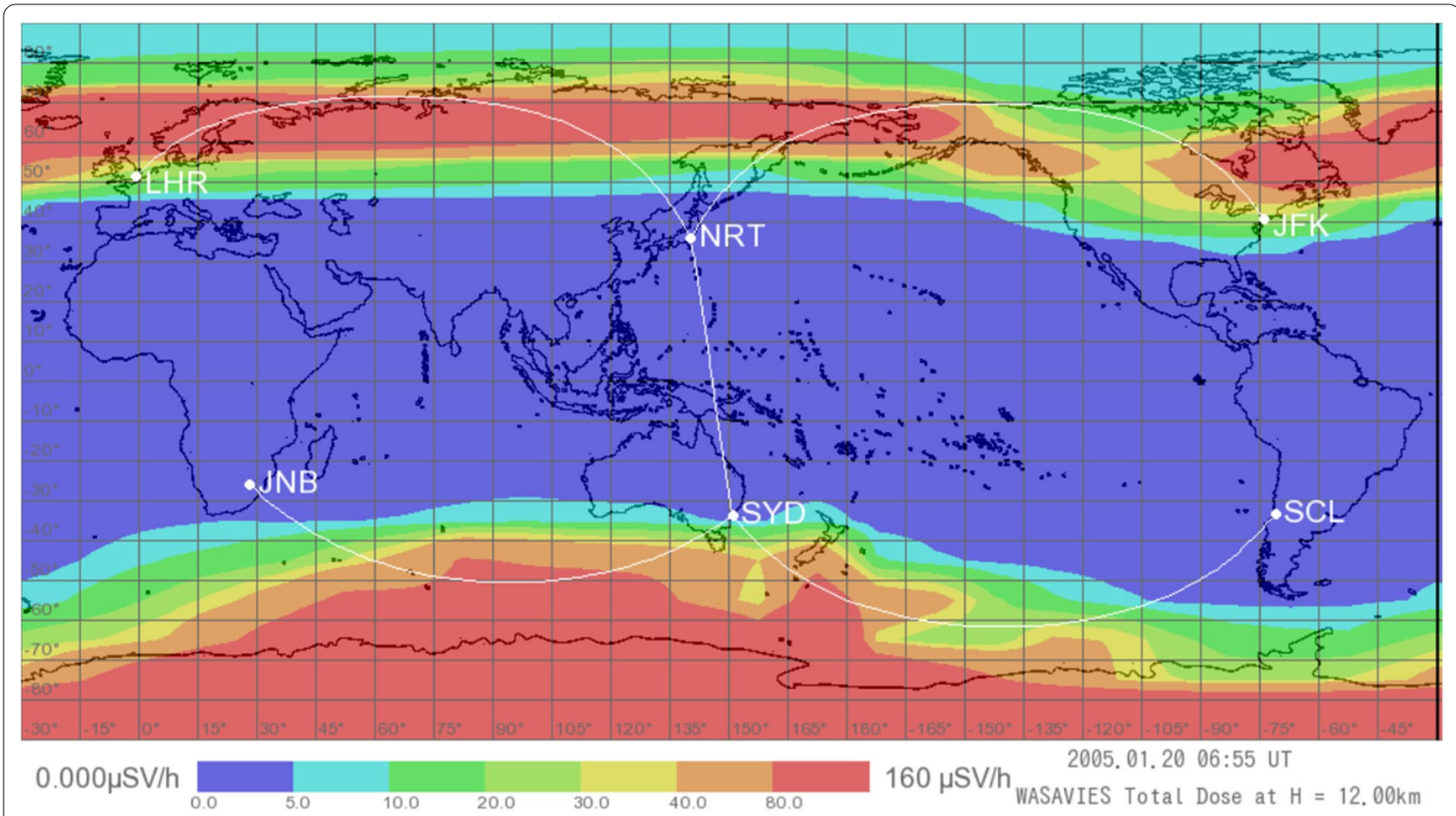

Fig. 4 Example of WASAVIES output. Colors indicate radiation doses at various locations

during a large solar flare calculated by WASAVIES will be used as mandatory information for aviation operation management by the International Civil Aviation Organization. An extended version of WASAVIES applicable to the Earth Orbit was also developed (Sato et al. 2019).

\section{Monitoring of the VHF anomalous propagation by the Es layer}

The Es layer could reflect the VHF radio waves to cause anomalous long-distance propagation. Because the frequency assignment in the VHF band including the aeronautical navigation band (from 108 to $118 \mathrm{MHz}$ ) considers only the propagation within the radio horizon, the anomalous propagation by the Es layer could cause radio interference.

We developed a network of receivers based on the software-defined radio technique to monitor signals of aeronautical radio navigation aids in 108 to $118 \mathrm{MHz}$ (Hosokawa et al. 2020). Since the locations and frequencies of aeronautical radio navigation aids are published as the aeronautical information publication, we can identify the source of anomalously propagating VHF radio waves. This, at the same time, allows us to detect the Es layer occurrence around the middle points between the signal sources and receivers. We found that anomalous propagation caused by the Es layer could occur in the aeronautical navigation band quite frequently, and the signal strength of the anomalous propagation could often exceed the limit required for airborne navigation receivers to work properly (Sakai et al. 2019). Furthermore, in some special geometries, the strength of anomalously propagating signals can be stronger than those of nearby stations, which may need to be considered in frequency assignment (Sakai et al. 2020).

With this VHF anomalous propagation monitoring network, information on the occurrence and location of the Es layer causing potential interference to the VHF radio systems can be provided in near real time. Furthermore, the VHF anomalous propagation occurrences could be predicted based on continuous monitoring of their occurrence and propagation.

\section{Discussion of the integration of space weather forecast models}

We collected information on models on the solar-terrestrial environment being developed in Japan to discuss the designs of integrated models for next-generation prediction models and/or further scientific models. A series of PSTEP science meetings "Prospects of Modeling Studies for Solar-Terrestrial Environment Prediction" (cohosted by Institute for Space-Earth Environmental Research (ISEE)) was organized four times (almost once 
per year) with the participation of experts from various backgrounds covering meteorology, space physics, solar physics, and astrophysics. We sorted out difficulties, challenges, priorities, and feasibilities related to the improvement of each model and model-model couplings toward the prediction model. Several efforts related to model coupling were accelerated via these meetings and achieved: e.g., between SUSANOO (heliosphere/solar wind) and radiation belt, between SUSANOO and GIC prediction model, and between solar spectral model and GAIA (see Sect. 3.3.2), and between the global magnetosphere MHD model at NICT and satellite charging analysis models (Nagatsuma et al. 2021). On the other hand, remained challenges for model coupling are, e.g., comprehensive coupling among magnetosphere-ionosphere-atmosphere, coupling from internal Sun to its surface and heliosphere, and global and local coupling of ionosphere models, because improvements of each model were more prioritized during the project. The information of models related to the PSTEP including the observational activities is summarized in Table 1 and the PSTEP web page (https://www.pstep.jp/model).

\section{Discussion of possible space weather disasters in Japan}

Space weather seldom has an impact on society, but when it does, the effects could be significant. It is necessary to estimate the quantitative scale and frequency of space weather disasters for proper preparation. To answer the requirement, we prepared a document named "Space Weather benchmarks on Japanese Society" (Ishii et al. 2021), which is the first in Japan, where the plausible space weather disasters in the country with scale and frequency are described and estimated from past studies. The information was compiled for domestic users of space weather forecasts for appropriate preparedness against space weather disasters. This paper gives a comprehensive summary of the survey, focusing on the fields of electricity, satellite operations, communication and broadcasting, satellite positioning usage, aviation, human space activity, and daily life on the Earth's surface, using the cutting-edge knowledge of space weather. Especially, we evaluated the social impact of space weather on electric power grid, satellite operation, aviation, telecommunication, broadcast, human space activity, and human activity on the ground (Ishii et al. 2021).

\section{Main results of A02 group: solar storms}

We aimed to improve our capability for predicting the solar-terrestrial environment by understanding the mechanism of solar explosions and the propagation of the consequent disturbances to the Earth using physics-based models. The primary objectives were as follows: (1) to develop a reliable scheme for predicting the occurrence of large-scale solar explosions by identifying its trigger mechanism using high-precision measurements of the magnetic fields on the Sun and advanced modeling based on physical principles; (2) to establish a methodology for forecasting solar storms that reach the Earth within several hours of the occurrence of the solar explosion by combining optical and radio observations and advanced modeling of the solar wind and CME, and (3) to form the basis for a next-generation space weather forecasting using a scheme for predicting the occurrence of large solar flares in a quantitative way from the synergetic combination of observations and modeling.

\section{Prediction of solar flares \\ A new physics-based model ( $k$-scheme) to predict large solar flares}

A new physics-based model called the $\mathrm{k}$-scheme was developed to predict large solar flares through the critical condition of a magnetohydrodynamic instability (Ishiguro and Kusano 2017) triggered by magnetic reconnection. Through the analysis of the largest (X-class) solar flares and the 198 active regions of the largest sunspots in Solar Cycle 24, it was demonstrated that the $\mathrm{k}$-scheme predicts not only the occurrence but also the precise location of imminent large solar flares (Fig. 5), with only small exceptions (Kusano et al. 2020). This study also found that the distribution of magnetic twist flux density near a magnetic polarity inversion line on the solar surface plays a crucial role in determining the onset and properties of solar flares. Some attempts have also been made to apply these findings to CME prediction (Lin et al. 2020, 2021).

The solar observation satellite "Hinode" has been operated during the period of the PSTEP project, giving its highest priority to flare observations. As a result, Hinode successfully recorded unique data for flares, including a series of X-class flares in September 2017. The acquired Hinode data have been primarily utilized for investigating trigger mechanisms of large flares with high-resolution, high-accuracy magnetic field vector maps at the photosphere. Bamba et al. (2017) identified the characteristic magnetic field structure on the magnetic polarity inversion lines and the preflare phenomena that triggered the large flares.

Magnetohydrodynamic simulations were conducted using the observed photospheric magnetic field as boundary conditions (Inoue et al. 2018a; b). The initial condition of each simulation was given a three-dimensional magnetic field that was numerically extrapolated from the photospheric field based on force-free approximation. The data-constrained simulations for the active regions 11,158 and 12,673 well reproduced the 


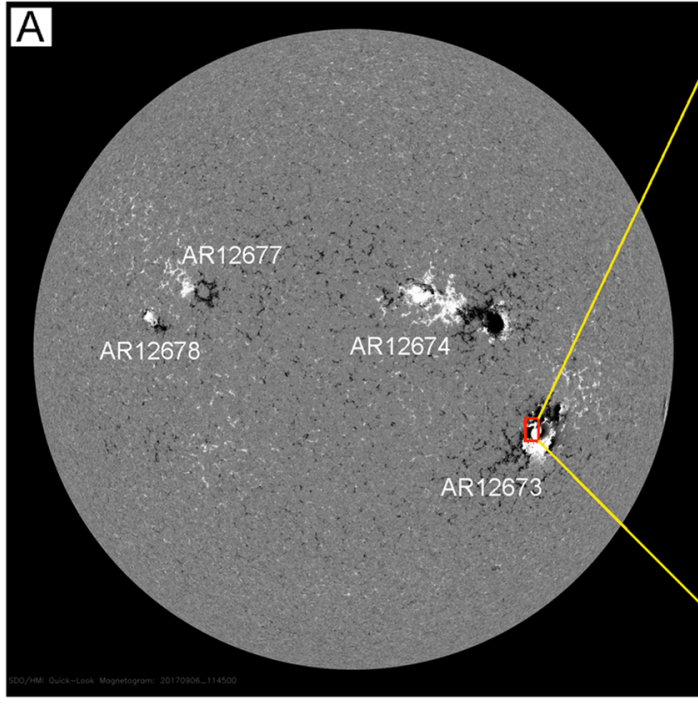

Magnetic field on the solar surface on September 6, 2017

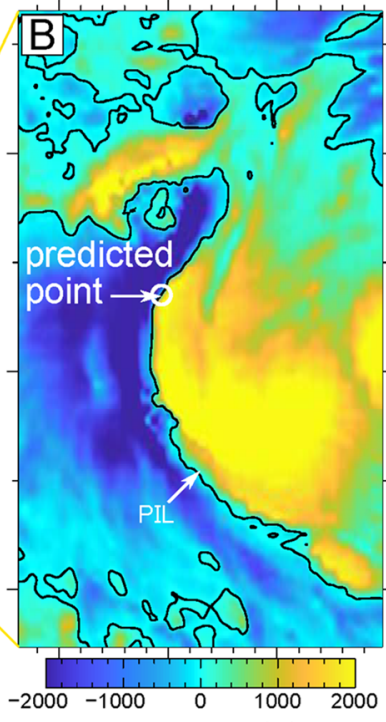

Magnetic field (G)

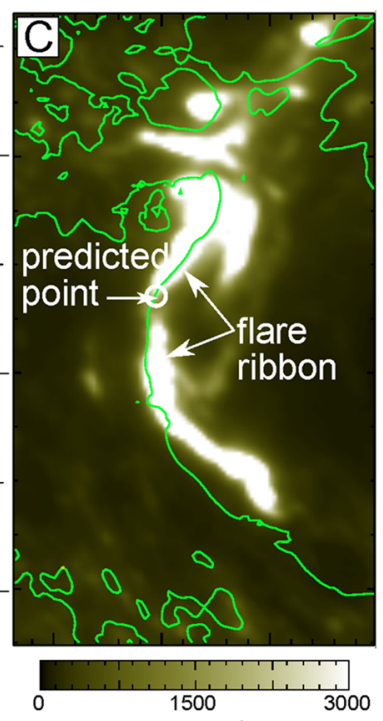

SDO/AIA $1600 \AA$ (DN)

Fig. 5 Magnetic field on the solar surface and the flare ribbon. A Shows the full-disk image of magnetic field component along the line of sight observed by the Solar Dynamics Observatory (SDO) satellite on 6 September 2017. White and black indicate the positive and negative components, respectively. Each active region (AR) number is noted. B, C are the zoom-in view of AR 12,673 for the vertical magnetic field on 08:48 UT observed by SDO/Helioseismic and Magnetic Imager (HMI) and the flare ribbon of the X9.3 flare at 08:59:26 UT observed by SDO/Atmospheric Imaging Assembly (AIA) $1600 \AA$. White circle represents the location where the k-scheme predicted a large flare can occur. B, C are reproduced from Fig. 3 of Kusano et al. 2020

characteristics of the large flares caused by each active region, in particular the spatial distributions and behaviors of the flare ribbons. It was also found that the erupting magnetic flux rope (MFR) is enhanced via magnetic reconnection and that the nonlinear interaction with magnetic reconnection is important for the acceleration of the erupting MFR.

Further developments for improving the accuracy of the coronal magnetic fields were conducted, i.e., Miyoshi et al. (2020) studied more realistic non-force-free field modeling of three-dimensional magnetic field in the solar corona, and Kawabata et al. (2020) incorporated the observed information of the chromospheric magnetic field in the boundary condition for extrapolating the coronal magnetic field. These results will contribute to the improvement of the accuracy of the coronal field modeling and eventually the flare prediction.

\section{Machine-learning algorithms for solar flare prediction}

Machine-learning algorithms were also applied to solar flare prediction. Nishizuka et al. (2018) developed a solar flare prediction model using deep neural networks, named Deep Flare Net (DeFN). The model can automatically detect active regions and predict the maximum class of flares occurring in the next $24 \mathrm{~h}$ after obtaining solar observation images. The model was trained using $3 \times 10^{5}$ magnetograms and coronal extreme ultraviolet
(EUV) images taken by the Solar Dynamic Observatories (SDO) satellite from 2010 to 2015, from which 79 physics-based features were extracted. DeFN was optimized and evaluated with the true skill statistic of 0.80 for $\geqq$ M-class flares and 0.63 for $\geqq C$-class flares. Among the 79 features, the most critical for predictions of flares are the history of flare occurrence, the information of magnetic polarity inversion lines, and the preflare brightening in the lower chromosphere. The reliability of DeFN was further improved by a new optimization method named as Deep Flare Net-Reliable (DeFN-R; Nishizuka et al. 2020), and it has been used in daily operation in NICT since January 2019 (Nishizuka et al. 2021).

\section{Benchmark test for operational solar flare forecasts}

In addition to the developments of new modeling for the flare prediction so far mentioned, a collaboration was made between the PSTEP and ISEE, Nagoya University, to organize the international workshop "Benchmarks for Operational Solar Flare Forecasts" and to publish a series of papers on the quantitative comparison between the 18 different methods cooperating with 16 institutes all over the world (Leka et al. 2019a, b; Park et al. 2020). In this workshop, multiple quantitative evaluation metrics were employed for the comparison, with the focus and discussion on evaluation methodologies given the restrictions of operational forecasting. They concluded 
that numerous methods performed consistently above the "no-skill" level, although which method scored top marks is decisively a function of flare event definition and the metric used; there was no single winner. With these works, they presented a well-defined and robust methodology for evaluating solar flare forecasting methods in both research and operational frameworks.

\section{Prediction of the occurrence of large-scale mass ejections}

As for the prediction of the occurrence of large-scale mass ejections from the Sun, some empirical methodologies were investigated. First, a precursor of filament eruption was found by using a newly developed Doppler-imaging instrument at Hida Observatory, i.e., an enhancement of plasma motions in a filament and slow rising motion of whole filament were observed prior to the onset of its eruption (Seki et al. 2017, 2019). Such behavior was also observed in 12 events of filament eruptions $0.2-24 \mathrm{~h}$ before the onset of violent eruption. Thus, the enhancement of internal plasma motions of filaments will provide a clue for predicting filament eruptions (Seki et al. 2019). The process that leads to filament eruption was also investigated by a numerical simulation, and the observed behavior before the violent eruption was well reproduced. The simulation showed that an enhancement of turbulent motions of prominence material is induced by the Kelvin-Helmholtz instability before the rapid growth of the instability.

\section{Observational aspect of the magnetic field in solar filaments}

The National Astronomical Observatory of Japan (NAOJ) has focused on the observational aspect of the magnetic field in solar filaments. Since its evolution causes the eruption of the filaments, the investigation of the magnetic field in filaments will open the way for the early prediction of the commencements of filament eruptions and that of the magnetic configuration of the erupted flux ropes, which occasionally hit the Earth's magnetosphere. To elucidate the general characteristics of the magnetic field in solar filaments, a statistical study of their average orientation was carried out. On the basis of data acquired with daily full-Sun, full-Stokes spectropolarimetric observations using the He I 1083.0-nm line by NAOJ, it was revealed that the average orientation of the magnetic field in filaments shows a systematic property depending on the hemisphere (Hanaoka and Sakurai 2017). This is the first confirmation of the hemispheric pattern in the filament magnetic field using direct measurements of the magnetic field in filaments.

On the other hand, the currently available observational data used above are not acquired with a sufficiently high-cadence to track the evolution of the magnetic field of the early stage of filament eruptions. To realize the high-cadence magnetic field observations, highperformance polarimetry in the near-infrared wavelengths employing a high-speed, large-format detector is required. Thus, the development of a new infrared camera, using a large-format array, HAWAII-2RG (H2RG) of Teledyne, was conducted (Hanaoka et al. 2020), and the synchronizing operation of the H2RGs under "fast readout mode" with a high-speed polarization modulation was successfully realized for the first time. The technology developed here will contribute to the launch of advanced solar polarimetry, which is required for nextgeneration space weather prediction.

\section{A new flare database and prediction of CME occurrence}

A new flare database, consisting of $211 \mathrm{M}$-class and X-class flares observed from 2006 to 2015 observed by the Hinode/X-ray telescope (XRT) and Geostationary Operational Environmental Satellite (GOES) was created. Through the comparison between the flare database and the database of CMEs observed by Large Angle and Spectrometric Coronagraph (LASCO) aboard the Solar and Heliospheric Observatory (SOHO), the Hinode team found that the sigmoidal structure in soft X-ray images will be a useful feature for predicting the CME occurrence in on-disk flare events (Kawabata et al. 2018). Furthermore, the team has developed an algorithm to automatically identify the appearance of sigmoidal structures in soft X-ray images. The automatic algorithm could open a new possibility to identify the potential occurrence of geoeffective CMEs in advance of flares, in addition to the observations of magnetic field configuration and the modeling of the circumstances preferable for triggering large flares.

\section{Real-time forecast of solar storms that reach the Earth Observation of high-speed eruptions from the sun}

To forecast the occurrence of CMEs and their arrival to the Earth by observing the initial phase of solar eruptions is the second primary objective of the group A02. It is known that many of CMEs are associated with prominence or filament eruptions, and the erupted filament is sometimes observed as the bright core of CMEs. In spite of the potential importance of the filament eruption for forecasting the occurrence and propagation of CMEs, none of the facilities for regular observation of solar eruption is able to capture the large line-of-sight velocity of erupting filaments because of a large Doppler shift of the observing spectral line.

To overcome this limitation and to establish a scheme for predicting the occurrence of CMEs that reach the Earth, a new solar imaging system to observe the threedimensional velocity of filament eruptions was installed at Hida Observatory under the PSTEP (Ichimoto et al. 
2017). The system (Solar Dynamics Doppler Imager, SDDI) takes full-disk solar images at multiple wavelengths around the $\mathrm{H} \alpha$ line at $6562 \AA$. Regular operation was started in May 2016, in which images at 73 wavelength positions spanning from $\mathrm{H} \alpha-9 \AA$ to $\mathrm{H} \alpha+9 \AA$ were obtained every $12 \mathrm{~s}$. The large dynamic range of the line-of-sight velocity measurements $\left( \pm 400 \mathrm{~km} \mathrm{~s}^{-1}\right)$ allows us to determine the real motions of erupting filaments in three-dimensional space (Fig. 6). Using 24 events of filament eruptions observed by SDDI in the period of May 1, 2016 to June 18, 2019, Seki et al. (2021) discovered that the product of maximum ascending velocity and length of erupting filament remarkably well correlates with the occurrence of CME. This finding shed a light on a new scheme for future use in operational space weather prediction.

\section{Metric solar radio burst observation}

One of the most important roles of metric solar radio burst observation for space weather forecasting is to immediately detect shock wave formation in the solar corona. Metric type II solar radio bursts are signals of coronal shock wave formation and can be used to estimate propagation speed of the shock wave. Estimated shock speed can be used as initial condition of shock wave propagation simulation in the solar wind. As shock wave can accelerate particles in the corona, it will be the signal of SEP production. Therefore, immediate detection of type II radio burst is fairly useful for space weather forecasting. NICT developed a solar radio spectrograph (Iwai et al. 2017) in YAMAGAWA radio observation facility to monitor solar radio burst. The observation officially started in September 2016. The observation data have been opened to the public by FITS format on the internet via https://solarobs.nict.go.jp/. By using the monitoring data, an automatic detection algorithm of type II solar radio burst is developed to issue an alert of shock wave formation. This algorithm is based on a fact that maximum intensity part of type II burst will become nearly straight line when the data are plotted on a time versus an inverse-frequency plane. Therefore, we applied an image processing technique to detect the straight line from dynamic spectrum images of time versus

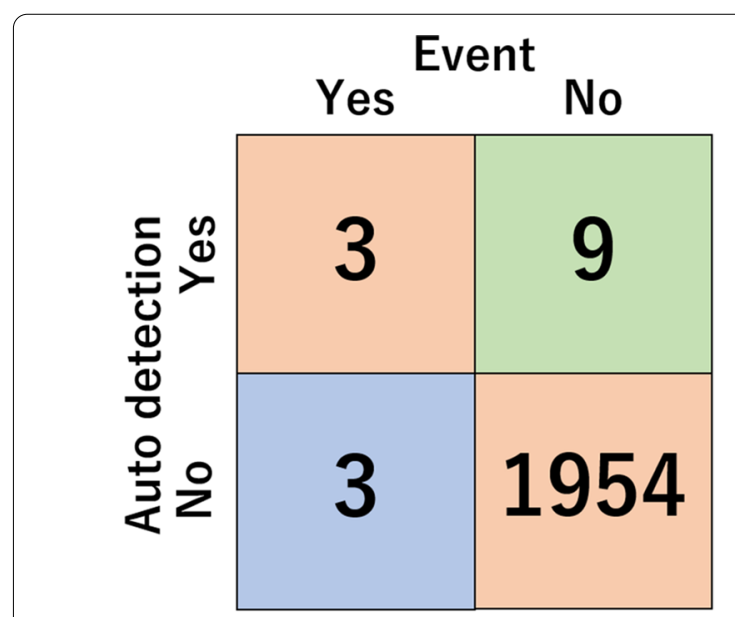

Fig. 7 Result of automatic detection of type II solar radio burst for almost 1 year data

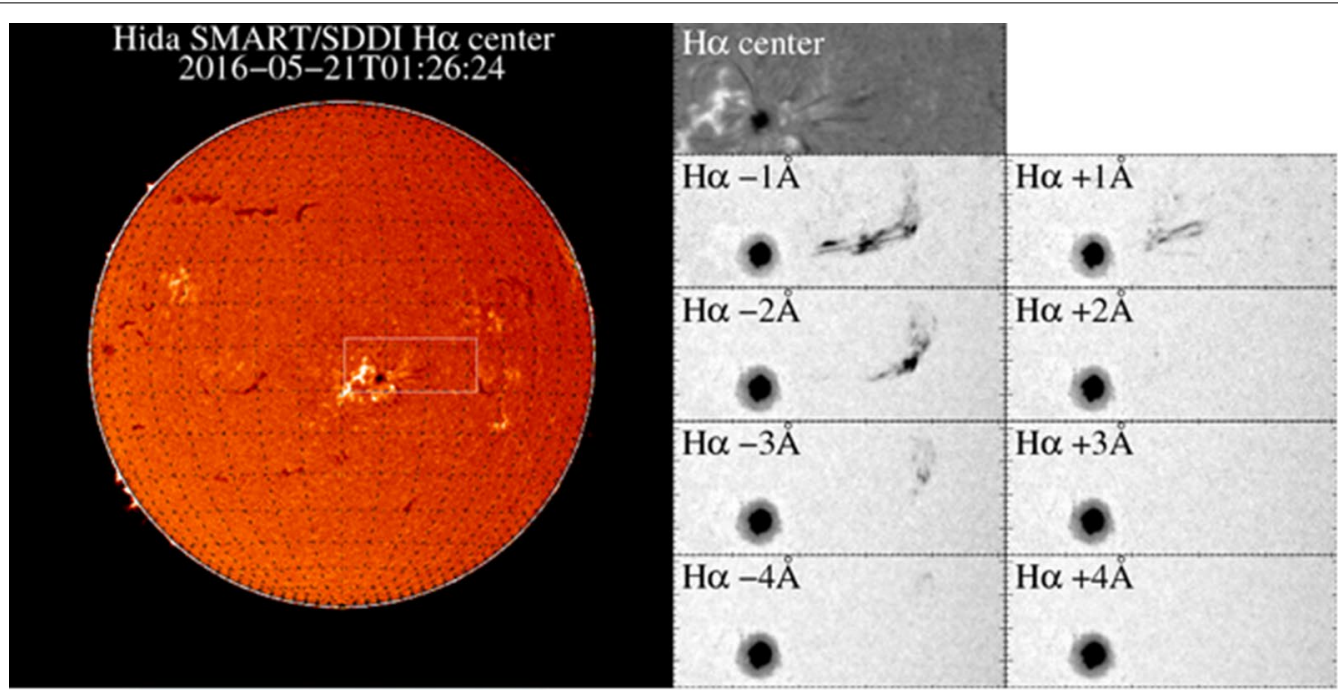

Fig. 6 Images of a filament eruption observed by SDDI at Hida Observatory on 2 May 2016. Left panel shows full-disk solar image taken in H-alpha line center. Right panels show partial images taken in different wavelengths around $\mathrm{H}$-alpha, where the bottom two images show components with a line-of-sight velocity of $-200 \mathrm{~km} / \mathrm{s}$ and $+200 \mathrm{~km} / \mathrm{s}$ 
inverse-frequency plane. After several preprocessings of the dynamic spectrum images with histogram equalization, filtering, and local peak detection, the probabilistic Hough transformation was used to detect straight line from the images. A detection performance was assessed by using the data for almost 1 year. The result is that the detection performance is fairly good, though false alarm occasionally occurs (see Fig. 7). The methodology for reducing the false alarm, such as using machine learning technique, is under investigation.

\section{SUSANOO-CME}

CMEs can be the main drivers of large-amplitude disturbances of the magnetosphere. The key parameters for such disturbances are southward interplanetary magnetic field (IMF) as well as high-speed and high-density solar wind, all of which can be brought by powerful CMEs. Shiota and Kataoka (2016) developed MHD simulation of the inner heliosphere called SUSANOO-CME, in which multiple CMEs including the internal magnetic field using modified spheromak magnetic field propagate in the background solar wind derived from the daily photospheric magnetic synoptic maps obtained in Global Oscillation Network Group Program. It was demonstrated that SUANOO-CME is capable of reproducing the arrival of a large amount of southward magnetic flux in high-speed such as the Halloween Storm in 2003.

For the purpose of application of SUSANOO-CME simulation into space weather forecast operation in NICT, Shiota (2020) have developed a system to automatically gather observational information as much as possible and to run ensemble SUSANOO-CME simulation based on the gathered observation. The system is planned to be used by nonscientist space weather forecasters, and therefore, the entire system is controlled by browser-based interfaces.

\section{Use of interplanetary scintillation data}

Interplanetary scintillation (IPS) is a radio-scattering phenomenon caused by density disturbances in the solar wind. CMEs in the interplanetary space can be detected by the enhanced IPS signatures during the observation of extra-galactic radio sources. ISEE, Nagoya University, has been observing IPS since 1980s using their own radio telescopes at $327 \mathrm{MHz}$. In the A02 project, Iwai et al. (2019) developed a CME arrival time forecasting system based on the IPS observational data and the above mentioned global MHD simulation, SUSANOO-CME. In this system, the initial speed of a CME is roughly derived from white-light coronagraph observations. Then, the propagation of the CME is calculated by SUSANOO-CME. The IPS response is estimated by the three-dimensional density distribution of the inner heliosphere derived from the MHD simulation. The simulated IPS data are compared with the actual IPS data observed by ISEE, Nagoya University.

The simulation run that best fits the IPS data was considered to be the most probable one, and its CME arrival time was automatically selected to the forecasting result. This system was validated using 12 halo CMEs. The average absolute arrival time error of the IPS-based SUSANOO-CME forecast was $\sim 5.0 \mathrm{~h}$, which is one of the most accurate predictions that has ever been validated. The real-time forecasting result for the same data set by another global MHD simulation, Enlil, archived in CCMC/NASA, was about $11.9 \mathrm{~h}$, and the result of the SUSANOO-CME simulations without IPS data, in which the initial CME speed was derived from whitelight coronagraph images, was $\sim 6.7 \mathrm{~h}$ (Iwai et al. 2021). This improvement using IPS data indicates that IPS can efficiently detect the CME fronts, and more IPS observation instruments should be developed for the future space weather forecast. This system has been partially installed in the space weather forecasting center in NICT and operated in real-time.

\section{Basis formation for a next-generation space weather forecasting \\ Formation of delta sunspots}

Observations revealed that the strongest solar flares in history emanate from complex-shaped active regions called the delta sunspots. However, their formation process has long been one of the key mysteries because the solar interior cannot be investigated from direct optical observations. Therefore, a series of MHD models that allow us to study the origin of such flare-prolific active regions have been developed. First, the emergence of magnetic flux tubes in a stratified atmosphere representing the solar interior with a variety of initial settings was simulated (Toriumi and Takasao 2017). As a result, it was found that the subsurface dynamics such as collision and twisting of flux tubes attribute complexity to the generated active regions, and these mechanisms well explained the observed characteristics (Toriumi et al. 2017). Then, by utilizing state-of-the-art radiative MHD code R2D2 (Radiation and Reduced speed of sound technique for Deep Dynamics), which simultaneously solves the realistic convection in the deep interior and smallscale surface granulation, the first-ever modeling of the spontaneous generation of delta sunspots through the coupling between magnetic fields and background turbulence was successfully performed (Toriumi and Hotta 2019). Elevated by large-scale upflows, the magnetic flux appears on the solar surface as two emerging bipoles, and the positive and negative polarities collide against each other to eventually create a pair of delta sunspots. 
The reproduced delta sunspots not only present sheared polarity inversion lines due to spot rotation driven by the Lorentz force but also form magnetic flux ropes in the atmosphere, all of which are the key ingredients of major flare eruptions. In this way, the series of modeling efforts deepen our understanding of flare occurrence and thus may contribute to the further establishment of flare predictions. This motivated us to publish the Living Reviews article, which summarizes the current understanding of the formation and evolution of flare-productive active regions that has been brought about through decades of observational and theoretical investigations (Toriumi and Wang 2019).

\section{$X$-rays and extreme ultraviolet emissions}

$\mathrm{X}$-rays and EUV emissions from solar flares rapidly change the physical composition of the Earth's thermosphere and ionosphere, thereby causing space weather phenomena such as communication failures. To find out the parameters that control the properties of flare emission, a statistical analysis on the observed flare emission data was performed to examine flare parameters related to the flare EUV spectra. From this study, it was found that the geometrical features of flare, such as flare loop length and ribbon distance, were found to be essential parameters for predicting flare emission duration (Nishimoto et al. 2020).

Then, to predict the effects of flare emissions on the Earth's upper atmosphere, a simple model based on the physical process of the flare loop (Kawai et al. 2020) is developed. In this model, the soft X-ray light curves observed during flare events were converted into EUV emission spectra using a one-dimensional hydrodynamic calculation and the CHIANTI atomic database. To verify the proposed model, the calculation results, and observed EUV spectra were compared in a statistical manner. The proposed model succeeded in reproducing the EUV flare time-integrated irradiance of the EUV lines, and the correlation coefficient between the proposed model and observations was higher than 0.8 for most of the EUV flare line rise time (Nishimoto et al. 2021).

An experiment was conducted to reproduce the variation of total electron content (TEC) in the Earth's atmosphere from the solar flare spectrum of the X9.3 flare on September 6, 2017. TEC distribution was obtained by inputting the solar flare spectrum obtained from the above study into GAIA. By comparing with the observed TEC distribution, it was found that the effective wavelength for increasing TEC is around $30 \mathrm{~nm}$ (Watanabe et al. 2021).

\section{Coordinated data analysis workshop on SEP}

The Coordinated Data Analysis Workshop (CDAW) is a community effort to promote the development and use of tools and techniques to analyze large volumes of joint datasets of selected solar-terrestrial events and to answer unsettled scientific questions. In order to establish longterm collaborations that can effectively address questions related to the SEP forecast, two CDAWs were held: one at Kyoto University in 2018 and another at Tohoku University in 2019. In the CDAWs, a dataset of 197 fast and wide coronal mass ejections (FW CMEs, $V \geq 900 \mathrm{~km} \mathrm{~s}^{-1}$, $W \geq 60^{\circ}$ ) was used to examine their SEP consequences. The characteristics of the FW CMEs and the associated flares and type II radio bursts were examined in conjunction with the SEP properties. Main results are as follows: a positive correlation (coefficient of 0.64 ) between the bandwidth of interplanetary type II radio bursts and the proton peak fluxes suggesting that the nonthermal electrons of type II bursts and the nonthermal ions of SEPs are generated by the same shock (Iwai et al. 2020), an isolation of short-duration $30-\mathrm{MeV}$ proton enhancements associated with the passage of interplanetary shocks or a turbulent magnetic cavity embedded in the ICME sheath region (Oka et al. 2021), and significant correlations between SEP events (i.e., the occurrence, peak flux, and arrival/rising time scales) and the CME speed and the source longitude; Kihara et al. (2020). These results will provide an important piece for building a sophisticated SEP forecast model in the frame of next-generation space weather forecasting.

\section{Main results of $\mathrm{A03}$ group: geospace dynamics}

The basic objectives of the A03 group were as follows: (1) to identify transient processes that cannot be captured by conventional radiation belt models and to improve the accuracy of predictions through comparison with Arase satellite observations; (2) to predict GICs by regionally coupled simulations and to improve the accuracy of predictions through verifications with observations, and (3) to predict geomagnetically induced currents in the vicinity of the geomagnetic field. We also predicted the probability of occurrence and arrival of ionospheric mesoscale disturbance phenomena such as plasma bubbles and improved the accuracy of the predictions based on the verification of observations. We have also investigated extreme events in geospace, radiation belts, ionospheric disturbances, and geomagnetic-induced currents from both observation and theoretical studies.

\section{Prediction of radiation belt variations Results from the Arase satellite data}

The research team conducted research about radiation belts and inner magnetosphere by data analysis of the 
Arase satellite and other satellite data and newly developed computer simulation. We proposed a new model for solar wind and radiation belt coupling (Miyoshi et al. 2016) and a model of cross energy coupling that is a fundamental process for electron accelerations and loss in the inner magnetosphere (Miyoshi et al. 2018). In this process, wave-particle interactions play an essential role for various coupling processes.

Using the data observed by the Japanese Arase satellite, we identified a rapid electron acceleration event that is caused by plasma waves, revealing that nonlinear waveparticle interactions play crucial roles in the acceleration of electrons in the geospace (Kurita et al. 2018a). We also successfully observed the direct evidence of pitch angle scattering by plasma wave-particle interactions by the Arase satellite (Kasahara et al. 2018). Moreover, we showed the first direct evidence of momentum transfer process between waves and particle by the newly developed algorithm WPIA (Shoji et al. 2017; Kitamura et al. 2018).

Using simultaneously observed data by the Arase and other satellites and by ground-based observation, we revealed the contribution of whistler mode chorus emissions (Kurita et al. 2018b), MHD waves (Teramoto et al. 2019), EMIC waves (Hirai et al. 2018; Nakamura et al. 2019), and electrostatic electron cyclotron harmonic waves (Fukizawa et al. 2018) to the loss process of energetic electrons.

\section{Computer simulations for wave-particle interactions}

We also reproduced the excitation process of whistler mode chorus and resonant scattering process of relativistic electrons by carrying out numerical simulations under a realistic plasma and magnetic field environment (Saito et al. 2016; Katoh and Omura 2016; Katoh et al. 2018). These simulation results clarified that nonlinear processes are essential in the generation process of chorus emissions and resultant acceleration and loss processes of radiation belt electrons (Fig. 8).

We developed real-time forecast of radiation belt electron flux by a code-coupling simulation of SUSNOAAsolar wind and radiation belt based on cooperation with A01. The real-time forecast has been operated for several years since 2016. The Brier Score (Brier 1950) that is a skill score for the forecast performance is 0.09 .

\section{Prediction of GICs}

\section{Measurements of GICs}

Direct measurements of GICs have been conducted in many countries, such as Finland (Pirjola 1985), Sweden (Wik et al. 2009), Spain (Torta 2014), Russia (Sakharov et al. 2009), Canada (Bolduc 2002), South Africa (Koen and Gaunt 2003), Australia (Marshall et al. 2013), New

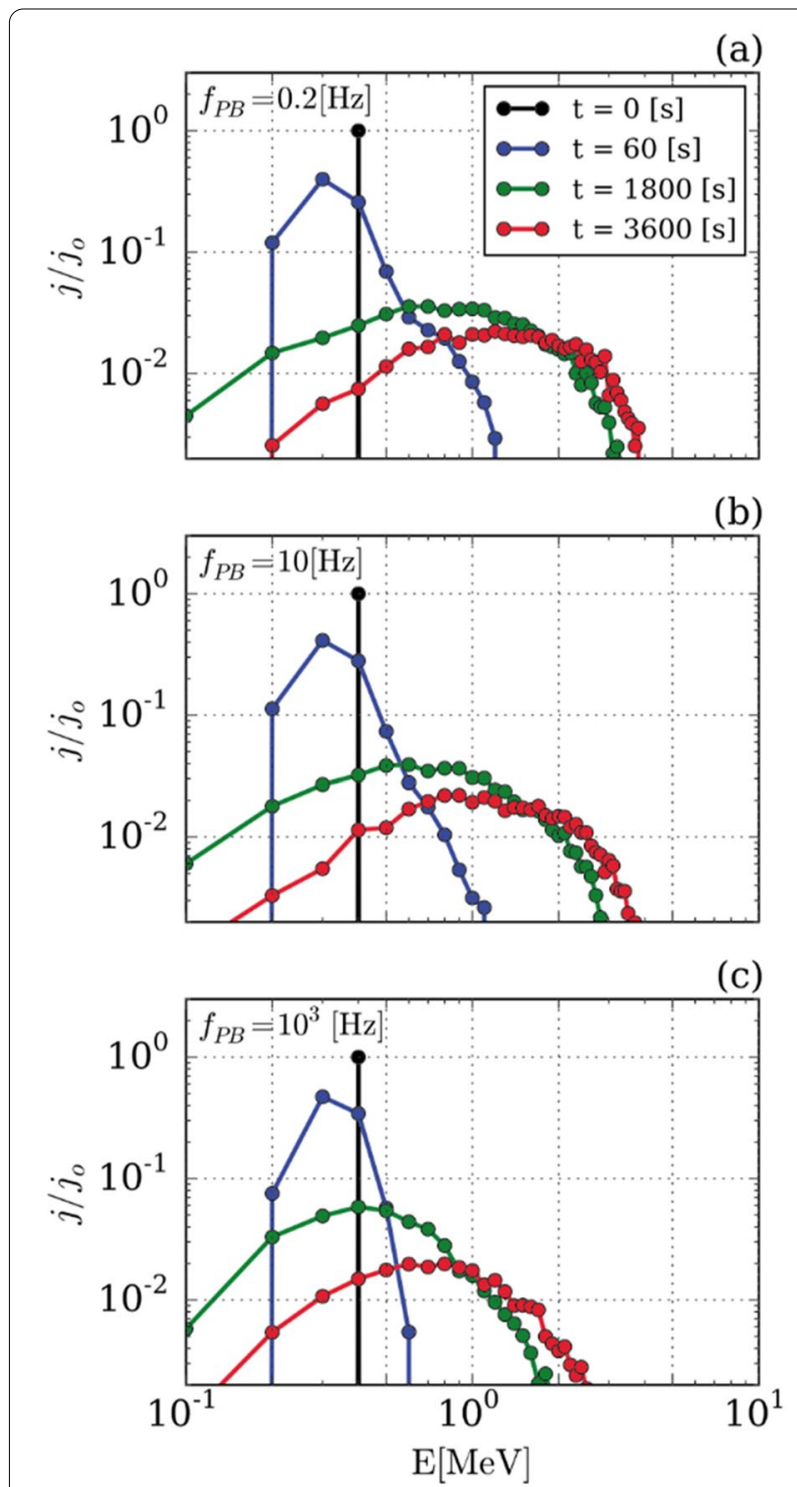

Fig. 8 Simulation results of the time evolution of the omnidirectional flux $j$ as a function of the kinetic energy of relativistic electrons under the presence of coherent chorus elements with rising tone (Saito et al. 2016). The wave phase variation of chorus elements is artificially distorted by the frequency (fPB) of (a) $0.2 \mathrm{~Hz}$, (b), $10 \mathrm{~Hz}$, and (c) $1000 \mathrm{~Hz}$, while the duration of chorus elements is assumed to be $1.25 \mathrm{~s}$ at the equator. These results clarified the significant effect of the phase trapping for the increase of the relativistic electron flux

Zealand (Marshall et al. 2012), China (Liu et al. 2009), and Japan (Watari et al. 2009). With the cooperation of power companies, we installed clamp-type current probes at four substations in Japan. The current was sampled every $0.1 \mathrm{~s}$ to capture rapid variations of GICs associated with storm sudden commencements (SSCs) or sudden impulses (SIs). The clock of the measurement system was synchronized with the GPS time information 
to guarantee the accuracy of absolute time. The data acquired at the four substations were automatically transferred in a real-time manner. The first measurement started in February 2017 and we decided to continue our measurements after this project (Watari et al. 2021).

\section{GIC modeling and simulations}

Two different methods have been used to model the GICs. The first one is to calculate the GICs directly with the aid of that transfer functions that describe the relationship between the GICs and the geomagnetic field (geoelectric field) (Ingham 1993; Pulkkinen et al. 2007). This method is applied to the substations at which the GICs are measured in advance. The other is to calculate the currents flowing in the network on the basis of Ohm's law and Kirchhoff's law for given transmission line resistance, ground resistance, and geoelectric field (Viljanen and Pirjola 1994). To model the geoelectric field, an impedance method (Kaufman and Keller 1981), a finite element method (Rodi 1976), a finite differential method (Mackie et al. 1994) have been used.

For the purpose of evaluating the GICs flowing in the Japanese power grid, we calculated the currents on the basis of Ohm's and Kirchhoff's laws. We developed simplified models for the transmission lines at $500 \mathrm{kV}$ (model A) and at voltage levels between 187 and $500 \mathrm{kV}$ (model B). Model A consists of 130 nodes (substations, generators, and switchyards) and 165 links (transmission lines). Model B consists of 602 nodes and 737 links. As for the geoelectric field, we also developed a threedimensional finite-difference time-domain (FDTD) simulation (Nakamura et al. 2018). The equivalent current inferred from the ground magnetic data was imposed on the topside boundary of the simulation box. Due to the source current, electromagnetic waves propagate downward, and penetrate into the ground. The ground resistance is nonuniform and determined on the basis of a global relief model and a sediment thickness model. As far as we know, this was the first model that calculates the global distribution of the GIC flowing in Japan. The results are shown in Fig. 9, together with the observed GIC described in Sect. 4.1.1. The correlation coefficient between the calculated GICs and the observed ones is 0.79 at a certain substation. The coefficient is 0.57 when we assumed a uniform ground resistance. This implies that realistic distribution of the ground resistance is necessary to improve the performance of prediction.

The input parameter for the FDTD simulation is the equivalent current, which can be estimated from the ground magnetic disturbances. Ground magnetic disturbances during substorms (Ebihara et al. 2014, 2019) and geomagnetic sudden commencements (Tanaka et al.

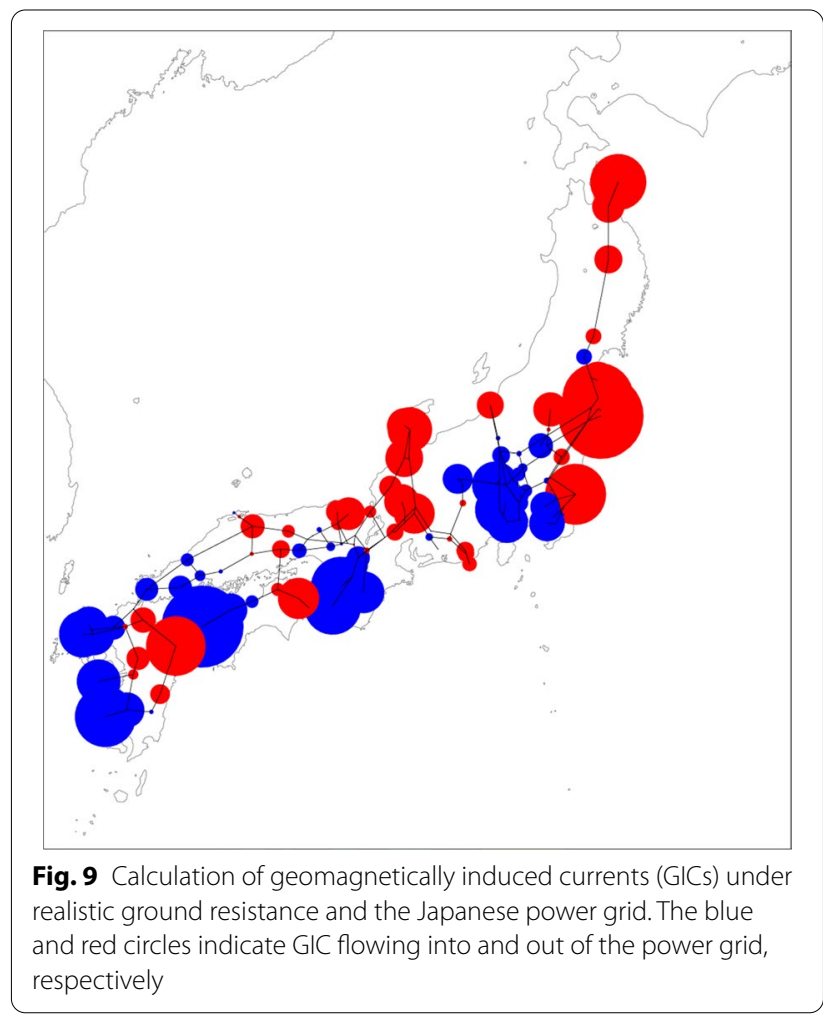

2019) can be numerically calculated by the global magnetohydrodynamics (MHD) simulation for given solar wind conditions.

\section{Prediction of ionospheric variations Observations of upper atmosphere}

Using all-sky airglow imagers and Fabry-Perot interferometers (FPIs) of optical mesosphere thermosphere imagers, new results have been obtained by the PSTEP project for characteristics of mesospheric gravity waves (AGWs) and MSTIDs. Tsuchiya et al. (2018) applied the three-dimensional fast Fourier transform (FFT) spectral analysis method to the airglow images obtained in Japan and found that the AGW propagation direction changed from southwestward to northeastward at Rikubetsu on the day of the zonal wind reversal associated with Sudden Stratospheric Warming (SSW). Based on long-term observations of AGWs, Takeo et al. (2017) and Tsuchiya et al. $(2019,2020)$ suggested that the longitudinal and seasonal differences of AGW propagation direction are caused by difference of wind filtering of AGWs by zonal mesospheric jet winds and by the locations of the observation sites relative to tropospheric wave sources.

Nakamura et al. (2017) compared thermospheric temperatures measured by FPIs at various latitudes and those obtained from GAIA, which is a self-consistent 
atmosphere-ionosphere coupled model, to evaluate the performance of the model in the thermosphere. It was found that the temperatures obtained by FPIs at middle latitudes are comparable to those obtained from GAIA. On the other hand, the GAIA temperature is $\sim 200 \mathrm{~K}$ lower than the FPI temperature in the auroral zone. This may be because the GAIA used in this study does not contain realistic thermospheric heating due to auroral energy input at high latitudes. At equatorial region, the difference of the GAIA and FPI temperatures is seen at around local midnight. This is possibly due to ambiguity of the location of midnight temperature maximum (MTM) in GAIA.

\section{Observations of equatorial ionosphere}

Dao et al. (2017) showed the generation of equatorial plasma bubbles around midnight associated with MTM using GNSS-TEC, airglow images, FPI thermospheric wind and temperature, and field-aligned irregularity (FAI) measurements over Indonesia. An enhancement of the magnetically equatorward thermospheric neutral wind was observed at the same time as an increase in the F-layer altitude at low latitudes in the night when the FAI was observed by a VHF radar around midnight. The observations suggest that convergence of equatorward neutral winds from the northern and southern hemispheres, which could be associated with MTM, could uplift the F-layer at low latitudes and increase the growth rate of the Rayleigh-Taylor instabilities, causing a rapid extension of plasma bubbles. Abadi et al. (2017), who statistically analyzed GNSS scintillation data obtained in Indonesia, found that scintillation occurs more frequently in the western azimuth than in the eastern azimuth from the ground-based receivers in March equinox, whereas such a feature is not discernible in September equinox. This result suggests that equatorial plasma bubbles are tilted westward in altitude and latitude more in March than in September equinox.

Moral et al. (2019) reported the first comparison of ground and satellite measurements of TIDs made by using the Challenging Minisatellite Payload satellite and a $630.0-\mathrm{nm}$ airglow imager at the equatorial station. They suggested that the MSTIDs observed at the equatorial region could be caused by AGWs. To identify the meteorological phenomena as the source of AGWs that cause ionospheric currents and electron density fluctuations as observed by low-altitude satellites, microbarometric, high-time resolution rainfall, and geomagnetic and GPS observations were conducted. The magnetic and electron density data provided by the ESA/Swarm satellite mission were also analyzed. From these analyses, it was suggested that the source of magnetic ripples and electron fluctuations observed in the ionospheric F-region along the satellite orbit is the acoustic mode waves caused by strong cumulous convection and strong rainfall in the troposphere (Aoyama et al. 2017).

\section{Observations of electrojet}

During the PSTEP project, a new FM-CW radar has been installed at Huancayo, Peru, in December 2018 and started the normal operation since March 2019. A comprehensive analysis of ground-based magnetometer network data (MAGnetic Data Acquisition System; MAGDAS: http://magdas2.serc.kyushu-u.ac.jp) and FM-CW observational data is carried out in order to understand the energy transfer and propagation process from polar to equatorial ionosphere in the terms of the coupling the solar-magnetosphere-ionosphere-atmosphere. Fujimoto et al. (2016) developed a new EE-index which can be separating the magnetic disturbances in the equatorial region into the global and local magnetic variations. They found that new EE-index allow us to be monitoring equatorial electrojet (EEJ) during both magnetically quiet and disturbed period and will be useful to compare the relationship between EEJ and growth/development of equatorial plasma bubbles (EPBs). Fujimoto et al. (2019) also analyze 20-year long-term semidiurnal lunar tidal variations of EEJ. They gave the evidence that the semidiurnal variations are completely different between the magnetic quiet and disturbed periods. This is the first time that the seasonal dependence of disturbance-time semidiurnal variation has been provided from the analysis of the new EE-index. Some important aspects of magnetosphere-ionosphere coupling at highlatitudinal regions are also found. For the generation of not only EEJ, but also auroral electrojet, the Cowling channel plays an important role. Due to this mechanism, inside a high-conductivity band, an intense electric current flow in the direction of the primary electric field by the secondary Hall current driven by the polarization electric fields. Yoshikawa and Fujii (2018) provides an extension of the theoretical model of the classical Cowling channel with respect to the following aspects: (1) taking into account the 3D nature of the ionosphere, and (2) considering finite length of the Cowling channel by introducing a conductance boundary not only at the meridional borders of the Cowling channel, but also at its zonal boundaries. Using this new model, they discuss current closure and the energy principle for evolution of the Cowling channel.

\section{Predictions using GAIA}

By comparing with the observation data of the ionosphere and thermosphere, GAIA was improved. Shinagawa et al. (2018) found that a high growth rate for the 


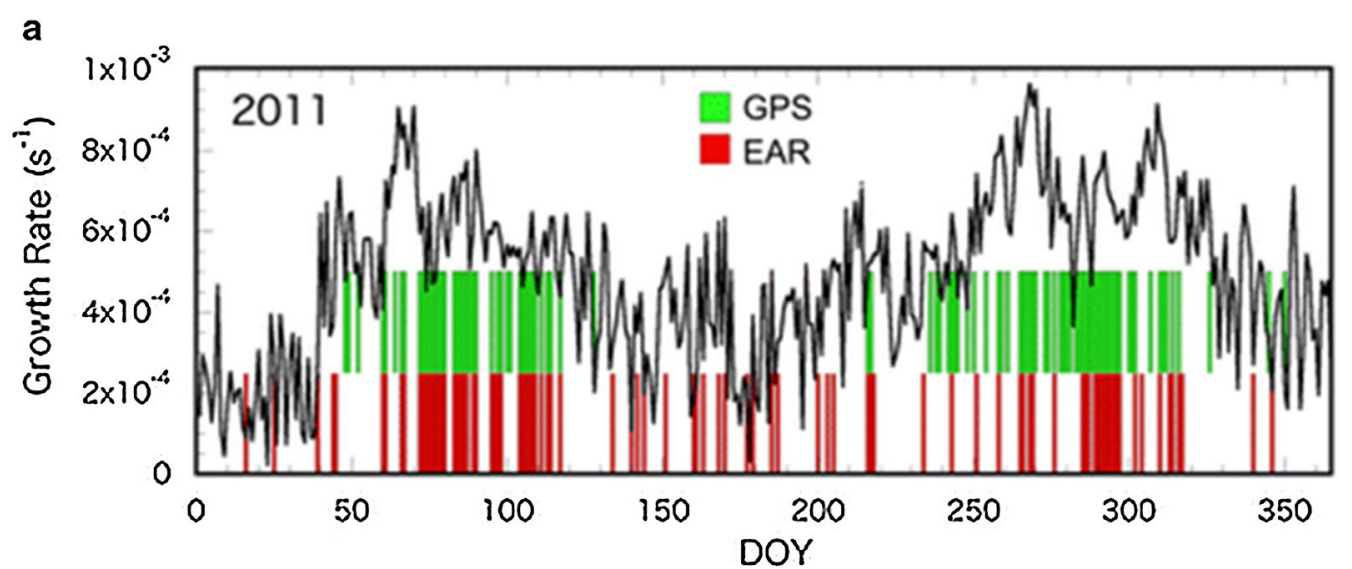

Fig. 10 Daily variations in R-T growth rate obtained with GAIA. The daily maximum values of the R-T growth rate (black line) are obtained with GAIA at $100^{\circ} \mathrm{E}$ for the years of 2011 . Vertical bars indicate the days when plasma bubbles are detected by EAR (red bar) and by GPS (green bar) (Shinagawa et al. 2018)

Rayleigh-Taylor instability obtained from GAIA coincides with the actual plasma bubble occurrence found by a VHF radar and GPS receivers in Indonesia (Fig. 10). By using a high-resolution numerical model of the ionosphere, Yokoyama et al. (2019) succeeded in reproducing the generation and development of plasma bubbles and showed that the vertical wind over the dip equator with an amplitude of speed as low as $5 \mathrm{~m} \mathrm{~s}^{-1}$ can effectively seed plasma bubbles. This result suggests that the vertical wind perturbations driven by AGWs should play an important role in seeding plasma bubbles.

Furthermore, Shinagawa et al. (2017) calculated vertical ion convergence induced by vertical wind shear originating from atmospheric waves, and they succeeded in reproducing local time and seasonal variations and global distribution of sporadic $E$ layer occurrence rate. Currently, GAIA is being operated in real-time by incorporating meteorological reanalysis data on the previous day below an altitude of $40 \mathrm{~km}$ via a nudging method to predict ionospheric phenomena which will occur in a few days. By comparing the observations, it was found that plasma bubble and sporadic $E$ occurrences can be predicted with an accuracy of more than $73 \%$ and $86 \%$, respectively. By including effects of the particle precipitations and the electric field from the magnetosphere to the ionosphere in GAIA, the ionospheric and thermospheric variations during magnetic disturbances have been reproduced in GAIA simulations. Including these two processes could reduce the discrepancy of thermospheric temperature at auroral zone between GAIA and observations described in Sub-Sect. 4.3.1.

\section{A04 group: solar cycle activity and impact on climate}

The basic goals of the A04 group were as follows: (1) to predict the next solar activity cycle through precise observation of solar activity and a new solar dynamo model and (2) to identify the impact of solar activity variations on climate through collaboration among solar physics, meteorology, and climatology. As a developmental goal, we have elucidated the mechanism of long-term solar activity variation on a multihundred-year scale and reproduced long-term variations using an Earth system model that incorporates solar influences.

\section{Prediction of the next solar cycle activity Variations of solar surface magnetic fields and simulation with a surface flux transport model}

The 24th solar cycle was the unique cycle with the fewest sunspots in the last 100 years, although the reason is still not clear. Using various methods, the 24th solar cycle prediction has been discussed (e.g., Pesnell 2016 and references therein). So far, the prediction schemes can be categorized into 6 (climatology, recent climatology, precursor, dynamo model, spectral, neural network). On the other hand, the prediction results differ for each method (see Fig. 3 in Pesnell 2016) and it is still needed to debate which is the best way to predict the next solar cycle. Predicting whether the activity of the next 25th solar cycle will be active again or decline is essential for predicting the solar-terrestrial environment in the coming decades. Thus, there is an urgent need to establish a method for predicting the next solar cycle. Recently, much attention has been paid to a method of predicting the next cycle activity by estimating the strength of a magnetic field in the polar region. In recent cycles, a positive correlation 
between the magnetic field strength in the polar region at the solar minimum and the activity in the next solar cycle has been confirmed.

To predict the next solar cycle activity, we developed a surface flux transport (SFT) model simulation code that calculates the temporal evolution of solar surface magnetic fields (Iijima et al. 2017, 2019). Iijima et al. (2017) found that the observed polar magnetic fields become approximately constant during the period of several years before each cycle minimum. This result allows us to predict the polar magnetic fields at the Cycle 24/25 minimum using the SFT model without introducing new flux emergence. The prediction result suggests that the activity of Cycle 25 will be even weaker than the current Cycle 24 (Fig. 11). Further, in order to improve the prediction accuracy, we have studied the temporal variation of the differential rotation and the poleward meridional circulation during Cycle 24 by a magnetic element feature tracking technique (Imada and Fujiyama 2018; Imada et al. 2020). In particular, during the declining phase of the cycle, the meridional flow of the middle latitude is accelerated from 10 to $17 \mathrm{~m} \mathrm{~s}^{-1}$, which is almost half of the meridional flow itself. The cross-equatorial transport of the net magnetic flux is important for estimating the polar magnetic field at the solar minimum. The crossequatorial transport of the net magnetic flux can be highly affected by the ratio between the meridional flow

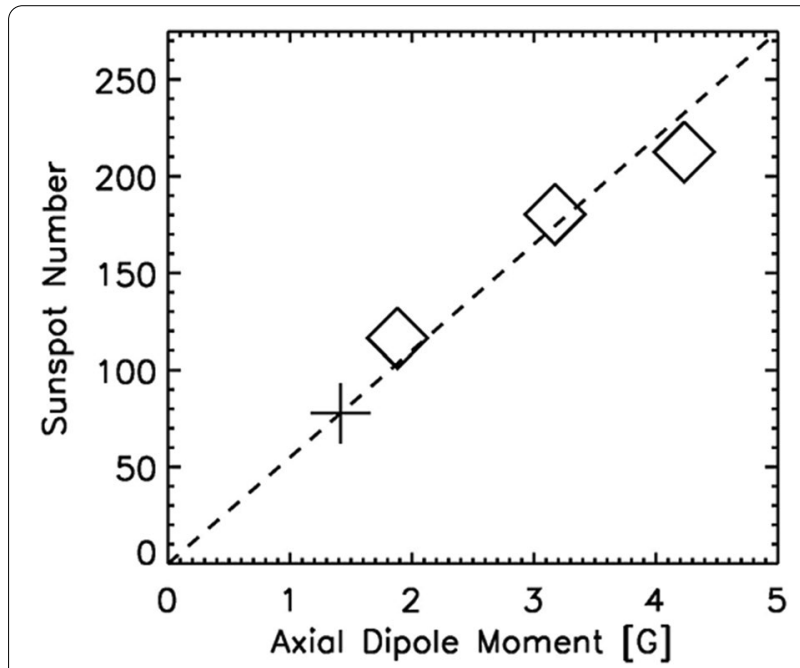

Fig. 11 Prediction of the maximum sunspot number for the next solar cycle (+) (lijima et al. 2017). Maximum value of the 13-month smoothed monthly total sunspot number in each sunspot cycle (Cycle 22, 23, and 24; diamond) and the predicted cycle amplitude in Cycle 25 (cross) as a function of the axial dipole moment at the previous minimum predicted from the magnetogram observed three years before the minimum. The least-square fit for Cycles 22, 23, and 24 that crosses the point of origin is shown as the dashed line. The correlation coefficient for Cycles 22, 23, and 24 is 0.99 and turbulent diffusion. Therefore, the temporal variation of the meridional flow velocity is an important parameter for determining the polar magnetic field at the solar minimum.

\section{High-resolution simulation of the solar global convection and the dynamo}

Our understanding of the solar dynamo, i.e., the generation mechanism of the magnetic field, was improved by higher resolution simulations. Previously, it was known that a large-scale magnetic field is destroyed by increasing the resolution, i.e., reducing the diffusivities, while the actual Sun has much smaller diffusivities. We improved our numerical method for reducing the numerical cost by using the reduced-speed-of-sound technique and carried out an unprecedentedly higher resolution simulation of the solar global convection and the dynamo by using the supercomputer KEI (Fig. 12). From a low-resolution to a middle-resolution computation, the result is consistent with the previous studies, i.e., the increase of the resolution causes the weakening of the large-scale magnetic field. In an unprecedentedly high-resolution computation, on the other hand, we discovered that the suppression of the destruction by a small-scale flow results in the amplification of a large-scale dynamo (Hotta et al. 2016).

This finding is crucial to understand the actual dynamo operating in the Sun. We also improved the numerical code to cover the whole convection zone from the base to the surface (Hotta et al. 2019). For the first time, we

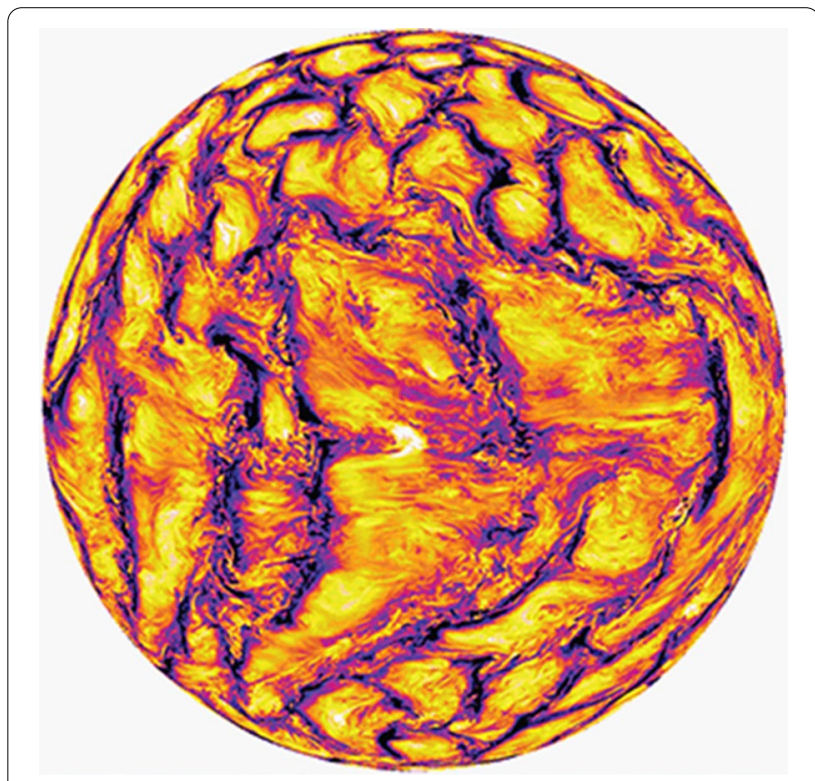

Fig. 12 The radial velocity around the top boundary. We see small-scale turbulent features as well as large-scale motion (Hotta et al. 2016) 
succeeded in carrying out such a computation and evaluated the influence of the near-surface layer to the deep convection zone. Furthermore, we carried out a simulation of the $\delta$ spot formation in collaboration with A02 group (Toriumi and Hotta 2019). We found that even with the surface phenomena such as the sunspot formation, we need to cover the full convection zone to understand the comprehensive process. We also prepared for the next-generation supercomputer FUGAKU. Using FUGAKU, we could extend our calculation domain closer to the surface and increase the resolution. These improvements lead to the reproduction of a more realistic solar situation.

\section{Ground-and satellite-based observations of solar magnetic fields}

We operated the infrared spectropolarimeter at the Mitaka campus of NAOJ over the whole period of the PSTEP and developed a data processing scheme to derive the magnetic field from polarization data (Sakurai et al. 2018). We also conducted mosaic observations to combine multiple scans of the Spectropolarimeter of the Solar Optical Telescope onboard Hinode to obtain magnetic field data covering wide regions. Our analysis of the wavenumber spectra of the current helicity using those mosaic observations was presented at the 10th Hinode Science Meeting held at Nagoya University in September 2016. The summary of the conference was published as a 118-page review paper, covering 11 years of science operation of Hinode (Hinode Review Team 2019). Sakurai (A04), Shiota (A01), Shimizu (A02), Toriumi (A02), and Imada (A04) were coauthors of this review paper. On the public relations and outreach activities, we published the second edition of "The Sun", volume 10 of the "Modern Astronomy Series" of Nippon Hyoronsha, edited by Sakurai, Kojima, Kosugi, and Shibata. The chapter on space weather has been substantially revised by Mitsue Den of NICT. Another activity was the production of a poster showing Solar Cycle 24 in various wavelength bands and instrumentation. This was one of the contributions of the A04 team to the PSTEP-4 International Symposium held at Nagoya University in 2020.

\section{Long-term variations of solar ultraviolet radiation}

We have been focusing on the long-term variation of solar ultraviolet (UV) radiation as one of the main effective factors of space climate. Solar UV radiation is mainly emitted from the chromosphere and is absorbed in the Earth's upper atmosphere. Astronomical Observatory of Kyoto University and NAOJ hold solar chromospheric imaging data in the Ca II K-line $(393.3 \mathrm{~nm})$ on glass plates from 1928 to 1969 and from 1917 to 1974 , respectively. These data are useful, since they directly provide the chromospheric features in the past. In the Ca II K-line images, we can identify various bright features, including plage, enhanced network, and quiet network. These bright features are the manifestation of the magnetic concentrations on the solar chromosphere, and they are also strongly correlated with the source of the solar UV radiation.

As part of the PSTEP, we digitally scanned the glass plate data, carefully calibrated them, and created the database (Kitai et al. 2014). By using the calibrated data, we succeeded to derive a butterfly diagram of the plage regions for the observing periods. During the PSTEP period, we also started international collaboration with India and analyzed the Ca II K-line data taken at Kodaikanal Observatory, which has even older data (from 1907). In particular, a new calibration method to convert the density on a Ca II K-line glass plate into the intensity was developed (Kakuwa and Ueno 2021) in order to quantitatively reproduce the UV intensity. We then performed a spectroscopic observation of the chromospheric Ca II K-line with the Domeless Solar Telescope (DST) at Hida Observatory, Kyoto University and showed the possibility of estimating the UV spectra from previous glass plate data by direct comparison with recent UV imaging data taken from satellites such as the SDO satellite.

Solar UV radiation has been sometimes substituted by microwave radiation, such as F10.7 $(2.8 \mathrm{GHz})$. F10.7 index is a good indicator of solar activity and has been recorded for over 70 years. Frequency ranges are sensitive to the solar chromosphere, where solar UV radiation is mainly emitted. Using the Nobeyama Radio Polarimeters at Nobeyama Solar Radio Observatory, NAOJ has also recorded microwave emission at multifrequency of 1, 2, 3.75, and 9.4 GHz (Shimojo et al. 2017). The longterm variations in those frequencies are similar with those of the F10.7 variation. Interestingly, the microwave intensities and the spectra at the solar minima of Cycles 20-24 did not show differences. The fact that the microwave radiations did not decline so much during the "anomalous Cycle" 23/24 minima (2008-2009) does not replicate the amount of decline in UV radiation expected from ionospheric variability and other factors affected by solar UV radiation. This divergence is a new mystery.

\section{Impact of solar activity variations on climate Simulations with MRI Earth System Model}

We developed the new Meteorological Research Institute Earth System Model version 2.0 (MRI-ESM2.0; see Yukimoto et al. 2019), reproducing realistic solar activity impacts on climate for the sixth phase of the Coupled Model Intercomparison Project (CMIP6; https://www. wcrp-climate.org/wgcm-cmip/wgcm-cmip6) experiments, which contributes to international assessments 
of climate change. In addition to the fine resolution of spectral solar irradiance (22 bands) as used in previous versions of the model MRI-CGCM3/MRI-ESM1 (e.g., Yukimoto et al. 2012), daily mean values of spectral solar irradiance instead of monthly mean values were applied in historical-related simulations (e.g., historical and AMIP experiments) in 1850-2014 (see SOLARISHEPPA recommendations for CMIP6; https://solarishep pa.geomar.de/cmip6). Effects of energetic particle precipitation (EPP) on atmospheric ozone chemistry were newly implemented. NOx and HOx production due to particle-induced ionization in the model domain was applied following the SOLARIS-HEPPA recommendations. Furthermore, NO molecular fluxes were prescribed at the model top $(0.01 \mathrm{hPa})$ as the upper boundary condition of the EPP effect on odd nitrogen (NOy). Ozone changes over the North Pole during the 2003 Halloween Storm was simulated by MRI-ESM2.0. Due to violent EPP, two spikes of large ozone depletions in the mesosphere occurred around October 28 and November 4 . While ozone reductions in the mesosphere recovered immediately, those in the upper stratosphere persisted throughout November. Even though the simulations did not perform initialization before this event, the behavior of ozone reductions is highly reproduced. Aside from the EPP effect, cloud radiative effect, and stratospheric circulations such as the quasi-biennial oscillation and sudden stratospheric warmings, which can modify solar activity impacts on climate, were also improved (e.g., Yukimoto et al. 2019; Naoe and Yoshida 2019).

\section{Simulations using GAIA}

In the mesosphere and thermosphere, some minor constituents, e.g., $\mathrm{CO}_{2}$ and $\mathrm{NO}$, contribute to the energy budget of the upper atmosphere through infrared radiations from them, while some, e.g., $\mathrm{H}_{2} \mathrm{O}$ and $\mathrm{HO}_{x}$, have impacts on ozone chemistry (ozone depletion) and production of the noctilucent cloud. Thus, it is necessary to understand the chemical and luminous phenomena in the terrestrial upper atmospheres to identify the behaviors of such minor constituents as well as variations of the major species. $\mathrm{NO}$ and $\mathrm{HO}_{x}$ are produced by precipitating particles in the polar mesosphere and thermosphere. The European incoherent scatter radar observations (e.g., on March 27, 2017) showed enhancements of the electron density in the 70 - to $100-\mathrm{km}$ altitude range, suggesting NO production in the polar region. Since the transport of minor species depends on atmospheric dynamics, e.g., global circulation, which shows seasonal and solar cycle variations, we should observe not only the minor species but also precipitating particles and neutral winds. Some modeling studies are also essential. We have investigated impacts of $\mathrm{NO}$ and $\mathrm{HO}_{x}$ on the thermosphere and ionosphere by using numerical models, e.g., GAIA (Jin et al. 2011). Effects of precipitating electrons and protons with energy of $0.1-1000 \mathrm{keV}$ on ionization of the polar upper atmosphere and production of $\mathrm{NO}$ and $\mathrm{HO}_{x}$ were modeled in the PSTEP.

\section{Effects of solar proton events on polar ozone using the MIROC chemistry-climate model}

Using the MIROC3.2 chemistry-climate model developed at the National Institute for Environmental Studies (Nakajima et al. 2020), we investigated the effects of giant solar proton events (Carrington Event on September 1, 1859) on polar ozone for several years. After validating the model with ozone changes after the 2003 Halloween Storm, the atmospheric composition in 1859 when the giant event occurred and the atmospheric composition in 2000 when the concentration of equivalent effective stratospheric chlorine reached the peak due to the release of CFCs were used for the calculation and results were compared. It was found that the influence of the proton events on the polar lower stratosphere ozone (ozone destruction) increases from spring to early summer due to the increase of chlorine and bromine compounds. We also found that the expansion of the impact is related to the interaction between the ozone, temperature, and westerly winds. These results indicate that future CFC regulation in accordance with the Montreal Protocol and its amendments could reduce the impact of future giant solar proton events on the ozone layer and climate.

\section{Passive-tracer transport experiments on the 775 AD Miyake Event using the EMAC model}

A series of passive-tracer transport experiments of $10 \mathrm{Be}$ produced in the 775 AD Miyake Event (Miyake et al. 2012), which is known as the largest solar high-energy particle event in the Holocene, were performed in order to clarify the seasonal dependence of the transport processes in the atmosphere (including the stratospheretroposphere exchange and surface deposition processes) and to limit the constraints on geological sample analysis such as ice cores. This study was made under international collaboration with Atmospheric Dynamics group of Free University of Berlin with their chemistry-climate model, EMAC (ECHAM/MESSy Atmospheric Chemistry) model, to perform an ensemble experiment of 10-year model integrations by injecting the tracer for every month of the year for ten times for different dynamical states.

To calculate the three-dimensional 10Be initial conditions, we used the latest version of the operational WASAVIES (Sato et al. 2018a) and evaluated the strength of the Miyake Event relative to the solar proton event of 2005 , which is the latest and perhaps best documented 
event. Recently obtained surface flux estimations of $10 \mathrm{Be}$ at four ice-core drilling sites in Greenland and Antarctica (two sites for each) were used for the evaluation of the relative strength. Moreover, the comparison of exponential decay in the first six years between the reconstructed ice-core data and the modeled data for each injection month concluded that the event most likely happened in early boreal springtime, in March. This study is a demonstration that such an extreme solar particle event as the Miyake Event is an ideal test case for solar-terrestrial physics, climate modeling, and geochronology. It can be used as a new modeling framework to evaluate model performances and to investigate atmospheric pathways with a state-of-the-art climate model, by comparing the model results to geological ice-core records. The results also provide new motivations in high-resolution geochronological proxy sample analyses regarding the seasonal timing of the event with contrast between the two hemispheres.

\section{Proxy-based observation for the little ice age}

Proxy-based observation of hydrological condition at the Little Ice Age has suggested that relative humidity in central Japan increases as solar activity decreases (Sakashita et al. 2017). Using the Japanese cedar collected at the Ise Grand Shrine, we investigated the variation of oxygen-18 in annually resolved tree-ring cellulose, which is an index of relative humidity. The growths of the trees are most active around June to July; therefore, oxygen-18 reflects the humidity around the rainy seasons. The reconstructed relative humidity indicated an increase toward the end of the Little Ice Age, but it shifted to drier condition since around $1850 \mathrm{CE}$. As directly observed, meteorological data suggest that relative humidity in Japan is strongly influenced by sea surface temperatures around the Philippines. It implies that low-latitude regions are the key area to understand the response of climate system to solar forcing.

To further understand the response of climate system to solar forcing, we also investigated the daily meteorological data in Japan (Miyahara et al. 2017a, b, 2018). Several researches have suggested that solar rotational period is often detected in meteorological phenomena such as cloud and thunderstorm activities. We analyzed thunderstorm record in Japan and found that solar rotational period becomes prominent as sunspot activity increases. Thunderstorm records over the 47 prefectures since $1989 \mathrm{CE}$ indicated that the signal of solar rotational period shows north-eastward migration, similar to the cloud activity pattern in summer in Japan. It again implies the importance of low-latitude regions as a possible recipient region of solar forcing.

\section{Investigation of long-term variations and predictions Proxy-based observation on past solar and cosmic ray variations}

Past solar and cosmic ray variations can be monitored by cosmogenic nuclides such as carbon-14 in tree rings and beryllium-10 in ice cores. In this project, we proceeded the annual-resolution measurements mainly around the grand solar minima. Previous studies have suggested that solar cycles are lengthened during the grand solar minima and that the lengthening might have even started a few cycles before the onset. High-precision carbon-14 data for around the onset of the Spoerer Minimum indicated that at least two cycles were lengthened before the onset (Moriya et al. 2019). Further analyses on solar cycles for various types of grand minima would shed light on what determines the duration of grand minima.

We also developed a new methodology for reconstructing past solar and cosmic ray variations using carbonate deposits called travertines (Xu et al. 2019; Miyahara et al. 2020). Travertines form clear annual layers and contain beryllium-10 produced by cosmic rays. Ice-core beryllium-10 data often accompany a few years of dating uncertainty; therefore, travertine beryllium-10 record may largely contribute to the reconstruction of annual event or of decadal-scale solar cycles in the past.

\section{Long-term solar variability based on contemporary historical documents}

The long-term solar variability in the past has been directly evaluated with sunspot group number, which have fairly good correlations with physical parameters such as sunspot area and radio emissions of $\mathrm{F}_{10.7}$. In this project, the long-term solar variability was analyzed with contemporary historical documents (Hayakawa et al. 2020a). Within the coverage of direct solar observations, we have two clear minima: The Maunder Minimum (1645-1715) and the Dalton Minimum (1797-1827). Analyses of these periods have been challenging, as the sunspot observations at that time are not abundant and have left significant uncertainty in their reconstructions. Consequently, the Dalton Minimum has been controversially understood, either as just a secular minimum vs one of grand minima.

In this context, Derfflinger's original sunspot records in 1802-1824 at the Kremsmünster Monastery have been subjected to thorough analyses (Hayakawa et al. 2020b), showing that the sunspot group number was revised and its long-term trend was updated, with omission of existing contaminations from the solar altitude observations. The sunspot positions were derived from his manuscript and shown in both solar hemispheres. These results are in contrast with extremely suppressed solar cycles, southward concentration of sunspot distribution during the 
Maunder Minimum (Hayakawa et al. 2020b). Moreover, total eclipse drawings in 1806 show significant coronal streamers unlike the apparent loss of coronal streamers during the Maunder Minimum (Hayakawa et al. 2020c). These results show that the mechanism of the Dalton Minimum is not probably something comparable to that of the Maunder Minimum but probably significantly different from that of the Maunder Minimum.

\section{Paleoclimate simulations using MRI Earth System Model}

A couple of paleoclimate numerical experiments were performed by using the Earth System Model of the Meteorological Research Institute (MRI-ESM1) to investigate the impact of interactive stratospheric ozone chemistry to the surface climate through the solar ultraviolet spectral modulation associated with variations in the Earth's orbital elements by the Milankovitch cycles. The time-slice results of interactive ozone chemistry runs for the mid-Holocene (6 kyr B.P.), and preindustrial (PI, 1850 AD) periods were compared with those of the corresponding runs in which the ozone distribution was prescribed to the PI level (Noda et al. 2017). The impact of the interactive ozone chemistry reveals a significant

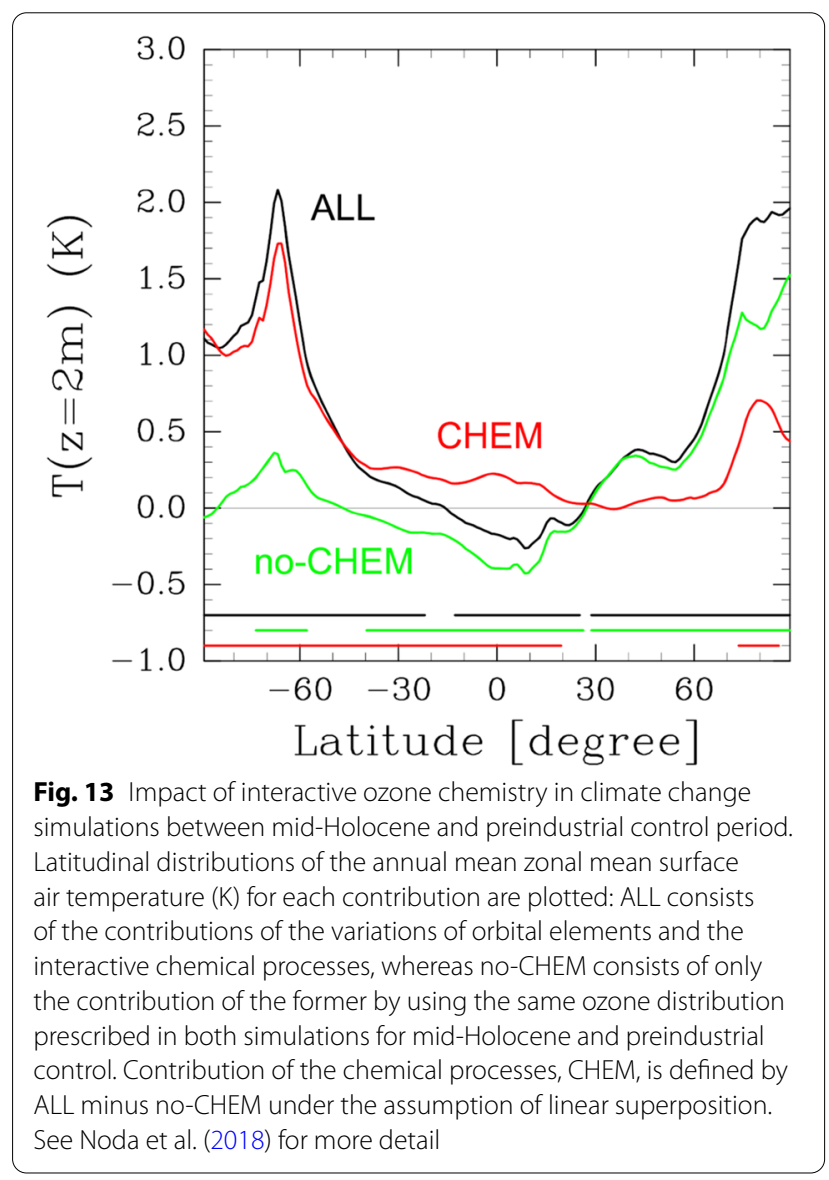

anomaly of up to $+1.7 \mathrm{~K}$ in the Antarctic region for the annual mean surface air temperature (SAT) as shown in Fig. 13. This impact on the surface climate is explained by a similar mechanism to the cooling influence of the Antarctic ozone hole but opposite in sign: Weakening of the westerly jet associated with the Southern Annular Mode provides weakening of equatorward ocean surface current, sea ice retreat, and then warm sea surface temperature (SST) and SAT. All the previous mid-Holocene runs with prescribed ozone distribution had a cold bias in SST when compared with geological proxy data, whereas the bias was reduced in our simulations by using interactive ozone chemistry.

Another series of numerical experiments on the Last Glacial Maximum (21 kyr B.P.) were also performed by using the same MRI-ESM1 to investigate the impact of interactive ozone chemistry (Noda et al. 2018). The impact was a significant warming of SAT, $+0.5 \mathrm{~K}(\sim 20 \%)$ in the tropics and up to $+1.6 \mathrm{~K}$ in high latitudes. The stronger mitigation of cooling in high latitudes is associated with sea ice retreat, which has the same sign to and is consistent with our mid-Holocene experiments. Most previous Last Glacial Maximum simulations with the prescribed ozone profile showed cold bias in the tropics compared with geological proxy data, whereas this bias is reduced in our simulations through the use of interactive ozone chemistry. Thus, it is recommended for these paleoclimate simulations that climate models should include interactive sea ice and ozone distribution that are consistent with paleosolar insolation.

\section{Concluding remarks}

The PSTEP has achieved many scientific research results and built several new schemes for space weather forecasting. The notable achievements of the PSTEP are listed as follows:

1. We developed the first-ever physics-based scheme ( $\mathrm{k}$-scheme) to predict large solar flares and discovered that the distribution of magnetic twist flux density near a magnetic polarity inversion line on the solar surface plays a crucial role in determining the onset and properties of solar flares.

2. We developed a new solar imaging system (SDDI) that can observe the three-dimensional velocity of filament eruption in the solar corona and discovered that the product of maximum ascending velocity and length of erupting filament remarkably well correlate with the occurrence of CME.

3. We developed a CME arrival time forecasting system based on the assimilation of the IPS observational data and the global MHD simulation, 
SUSANOO-CME, and verified that this system significantly improves the CME arrival prediction.

4. We proposed a new model for solar wind and radiation belt coupling that considers wave-particle interactions of various nonlinear processes.

5. We developed a three-dimensional simulation model to describe the propagation of electromagnetic fields in the atmosphere, the ground, and the transmission lines to predict the GIC during space weather disturbing phases and evaluated the predictability by measuring the GIC in cooperation with Japanese power companies.

6. We discovered that the atmosphere-ionosphere coupling model could predict the possibility of equatorial plasma bubbles and sporadic $\mathrm{E}$ layer occurrence.

7. We developed a high-resolution simulation of the solar global convection and the dynamo and discovered the role of small-scale magnetic field to produce large-scale magnetic field in turbulence. We also predicted the solar dynamo activity of the next solar cycle (Cycle 25) based on a SFT model.

8. We investigated the causal relationship between EPP on the atmosphere and ozone reduction in the upper stratosphere through NOx and HOx production due to particle-induced ionization.

9. We established "Space Weather Users Committee Meeting" for discussing the needs-seeds matching in space weather services.

10. We developed new systems of space weather forecast: the radio propagation simulator (HF-START), the space environment customized risk estimation for satellites (SECURES), and the estimation system of satellite charging, and the warning system for aviation exposure to solar energetic particles (WASAVIES).

11. We published Japan's first space weather benchmarks to evaluate a space weather disaster's social impact.

The PSTEP produced many research results and finished in March 2020. Through the PSTEP, we have demonstrated that physics-based predictions can work well and be very informative for predicting and understanding solar-terrestrial environment dynamics. However, research and development to apply these results for daily space weather forecasts remain as future tasks. For instance, we need higher speed computing to use the physics-based flare prediction ( $\mathrm{K}$-scheme) to the daily operational forecast of solar flares, and we have to create a network system which can connect the real-time observations and the physics-based prediction models. We should continue our efforts to do so. The activities of PSTEP have created the basis for the next-generation space weather forecast and demonstrated how important is the synergistic development between operational forecast and basic research of the solar-terrestrial environment for the future study of space weather.

\section{Abbreviations}

AGW: Atmospheric gravity waves; CDAW: Coordinated Data Analysis Workshop; CFC: Chlorofluorocarbon; CGCM: Coupled General Circulation Model; CME: Coronal mass ejection; CMIP: Coupled Model Intercomparison Project; DeFN: Deep flare net; Dst: Disturbance storm-time; EEJ: Equatorial electrojet; EMAC: ECHAM/MESSy atmospheric chemistry; EPB: Equatorial plasma bubbles; EPP: Energetic particle precipitation; ESA: Earth Sensor Assembly; ESA: European Space Agency; ESD: Electrostatic discharge; ESM: Earth System Model; EUV: Extreme ultraviolet; FAl: Field-aligned irregularity; FM-CW: Frequency modulated continuous wave; FPI: Fabry-Perot interferometer; GAIA: Groundto-topside model of atmosphere and ionosphere for aeronomy; GEONET: GPS network in Japanese area; GIC: Geomagnetically induced current; GLE: Ground level enhancement; GNSS: Global Navigation Satellite System; GOES: Geostationary Operational Environmental Satellite; GPS: Global Position System; H2RG: HAWAll-2RG; HF-START: HF simulator targeting for all users' regional telecommunications; IMF: Interplanetary magnetic field; IPS: Interplanetary scintillation; ISEE: Institute for Space-Earth Environmental Research; LANL: Los Alamos National Laboratory; LASER: Low-cost Amateur receiver System Employing RTL-SDR; MAGDAS: MAGnetic Data Acquisition System; MHD: Magnetohydrodynamics; MIROC: Model for Interdisciplinary Research on Climate; MPA: Magnetospheric Plasma Analyzer; MRI: Meteorological Research Institute; MSTID: Medium-scale traveling ionospheric disturbance; MTM: Midnight temperature maximum; MUSCAT: Multi-utility spacecraft charging analysis tool; NAOJ: National Astronomical Observatory of Japan; NICT: National Institute of Information and Communications Technology; PHITS: Particle and Heavy lon Transport code System; PI: Preindustrial; PSTEP: Project for Solar-Terrestrial Environment Prediction; R2D2: Radiation and reduced speed of sound technique for deep dynamics; SAT: Surface air temperature; SDDI: Solar dynamics Doppler imager; SDO: Solar Dynamic Observatories; SECURES: Space environment customized risk estimation for satellites; SEP: Solar energetic particle; SFT: Surface flux transport; SI: Sudden impulse; SOLARIS-HEPPA: Solar influences for SPARC (stratospheric processes and their role in climate)-high energy particle precipitation in the atmosphere; SPIS: Spacecraft plasma interaction system; SSC: Sudden storm commencement; SST: Sea surface temperature; SSW: Sudden stratospheric warming; SUSANOO: Space weather forecast Usable System Anchored by Numerical Operations and Observations; TEC: Total electron content; TID: Traveling ionospheric disturbance; UV: Ultraviolet; WASAVIES: Warning system for aviation exposure to solar energetic particles; WPIA: Wave particle interaction analyzer.

\section{Acknowledgements}

This work was supported by JSPS KAKENHI Grant, 15H05812, 15K21709, 15H05813, 15H05814, 15H05815, $15 \mathrm{H} 05816$.

\section{Authors' contributions}

All authors contributed to write each subsubsection or paragraph(s) of the manuscript, and the first five authors (KK, Kl, MI, YM, and SY) made arrangements of those in each section. All authors read and approved the final manuscript.

\section{Funding}

Grant-in-Aid for Scientific Research (KAKENHI) on Innovative Areas from the Ministry of Education, Culture, Sports, Science and Technology of Japan for "Solar-Terrestrial Environment Prediction as Science and Social Infrastructure (PSTEP)" is the main source of funding, under the Grant numbers of $15 \mathrm{H} 05812$, 15K21709, 15H05813, 15H05814, 15H05815, and 15H05816.

\section{Availability of data and materials}

This is a summary report of research collaborations under PSTEP. If necessary, all datasets used for PSTEP research would be provided by the corresponding authors of each cited paper. 


\section{Declarations}

Ethics approval and consent to participate

Not applicable.

\section{Consent for publication}

Not applicable.

\section{Competing interests}

The authors declare that they have no competing interests.

\section{Author details}

1 Institute for Space-Earth Environmental Research (ISEE), Nagoya University, Nagoya 464-8601, Japan. ${ }^{2}$ Astronomical Observatory, Kyoto University, Kyoto 606-8502, Japan. ${ }^{3}$ National Institute of Information and Communications Technology (NICT), Koganei, Tokyo 184-8795, Japan. ${ }^{4}$ Institute for Space-Earth Environmental Research (ISEE), Nagoya University, Nagoya 464-8601, Japan. ${ }^{5}$ Institute for Liberal Arts and Sciences, Kyoto University, Kyoto 606-8501, Japan. ${ }^{6}$ National Institute for Environmental Studies, Tsukuba, Ibaraki 305-8506, Japan. ${ }^{7}$ Astronomical Observatory, Kyoto University, Kyoto 606-8502, Japan. ${ }^{8}$ Research Institute for Sustainable Humanosphere (RISH), Kyoto University, Uji, Kyoto 611-0011, Japan. ${ }^{9}$ Seikei University, Musashino, Tokyo 180-8633, Japan. ${ }^{10}$ University of Hyogo, Himeji 671-2280, Japan. ${ }^{11}$ National Astronomical Observatory of Japan (NAOJ), Mitaka, Tokyo 181-8588, Japan. ${ }^{12}$ University of Electro-Communications, Chofu, Tokyo 182-8585, Japan. ${ }^{13}$ Chiba University, Chiba 263-8522, Japan. ${ }^{14}$ Kyoto University, Kyoto 606-8502, Japan. ${ }^{15}$ National Institute for Polar Research (NIPR), Tachikawa, Tokyo 190-8518, Japan. ${ }^{16}$ Graduate School of Science, Tohoku University, Sendai 980-8578, Japan. ${ }^{17}$ National Institute of Information and Communications Technology (NICT), Koganei, Tokyo 184-8795, Japan. ${ }^{18}$ Japan Aerospace Exploration Agency (JAXA), Tsukuba, Ibaraki 305-8505, Japan. ${ }^{19}$ Institute of Space and Astronautical Science/Japan Aerospace Exploration Agency (ISAS/JAXA), Sagamihara, Kanagawa 252-5210, Japan. ${ }^{20}$ Musashino Art University, Kodaira, Tokyo 187-8505, Japan. ${ }^{21}$ Department of Earth and Planetary Sciences, Kyushu University, Fukuoka 819-0395, Japan. ${ }^{22}$ National Institute of Information and Communications Technology (NICT), Koganei, Tokyo 184-8795, Japan. ${ }^{23}$ Graduate School of Engineering, Chiba University, Chiba 263-8522, Japan. ${ }^{24}$ National Institute of Maritime, Port and Aviation Technology, Electronic Navigation Research Institute (ENRI), Chofu, Tokyo 182-0012, Japan. ${ }^{25}$ Japan Atomic Energy Agency (JAEA), Tokai-mura, Ibaraki 319-1195, Japan. ${ }^{26}$ Hida Observatory, Kyoto University, Kurabashira, Kamitakara, Gifu 506-1314, Japan. ${ }^{27}$ National Defense Academy of Japan, Yokosuka, Kanagawa 239-8686, Japan. ${ }^{28}$ The Catholic University of America, Washington, DC 20064, USA. ${ }^{29}$ Meteorological Research Institute, Tsukuba, Ibaraki 305-0052, Japan. ${ }^{30}$ International Center for Space Weather Science and Education, Kyushu University, Fukuoka 819-0395, Japan.

\section{Received: 17 December 2020 Accepted: 19 July 2021}

\section{Published online: 05 August 2021}

\section{References}

Abadi P, Otsuka Y, Shiokawa K, Husin A, Liu H, Saito S (2017) Equinoctial asymmetry in the zonal distribution of scintillation as observed by GPS receivers in Indonesia. J Geophys Res Space Physics 122:8947-8958. https://doi.org/10.1002/2017JA024146

Aoyama T, Iyemori T, Nakanishi K (2017) Magnetic ripples observed by Swarm satellites and their enhancement during typhoon activity. Earth Planets Space 69:89. https://doi.org/10.1186/s40623-017-0679-2

Bamba Y, Inoue S, Imada S (2020) Intrusion of magnetic peninsula toward the neighboring opposite-polarity region that triggers the largest solar flare in Solar Cycle 24. Astrophys J 894:29. https://doi.org/10.3847/1538$4357 / \mathrm{ab} 85 \mathrm{ca}$

Bamba Y, Kusano K (2018) Evaluation of applicability of a flare trigger model based on a comparison of geometric structures. Astrophys I 856:43. https://doi.org/10.3847/1538-4357/aaacd1

Bamba Y, Lee K-S, Imada S, Kusano K (2017) Study on precursor activity of the X1.6 flare in the great AR 12192 with SDO, IRIS, and Hinode. Astrophys J 840:116. https://doi.org/10.3847/1538-4357/aa6dfe
Bolduc L (2002) GlC observations and studies in the Hydro-Quebec power system. J Atmos Solar Terr Phys 64(16):1793-1802. https://doi.org/10. 1016/S1364-6826(02)00128-1

Bowman GG (1960) Some aspects of sporadic E at mid-latitudes. Planet Space Sci 2(4):195-202. https://doi.org/10.1016/0032-0633(60)90016-7

Bowman GG (1968) Movements of ionospheric irregularities and gravity waves. J Atmos Terr Phys 30(5):721-734. https://doi.org/10.1016/S00219169(68)80028-5

Brier GW (1950) Verification of forecasts expressed in terms of probability. Mon Weather Rev 78:1-3. https://doi.org/10.1175/1520-0493(1950)078\% 3c0001:VOFEIT\%3e2.0.CO;2

Dao T, Otsuka Y, Shiokawa K, Nishioka M, Yamamoto M, Buhari SM, Abdullah M, Husin A (2017) Coordinated observations of post-midnight irregularities and thermospheric neutral winds and temperatures at low latitudes. J Geophys Res 122:7504-7518. https://doi.org/10.1002/2017JA024048

Ebihara Y, Tanaka T, Kamiyoshikawa N (2019) New diagnosis for energy flow from solar wind to ionosphere during substorm: global MHD simulation. J Geophys Res Space Physics 124:360-378. https://doi.org/10. 1029/2018JA026177

Ebihara Y, Tanaka T, Kikuchi T (2014) Counter equatorial electrojet and overshielding after substorm onset: Global MHD simulation study. J Geophys Res 119:7281-7296. https://doi.org/10.1002/2014JA020065

Fujimoto A, Uozumi T, Abe S, Matsushita H, Imajo S, Ishitsuka JK, Yoshikawa A (2016) Long-term EEJ variations by using the improved EE-index. Sun Geosphere 11(1):37-47

Fujimoto A, Yoshikawa A, Uozumi T, Abe S (2019) Seasonal dependence of semidiurnal equatorial magnetic variation during quiet and disturbed periods. E3S Web of Conferences, 127. https://doi.org/10.1051/e3sconf/ 201912702025

Fukizawa M, Sakanoi T, Miyoshi Y, Hosokawa K, Shiokawa K, Katoh Y, Kazama Y, Kumamoto A, Tsuchiya F, Miyashita Y, Tanaka Y-M, Kasahara Y, Ozaki M, Matsuoka A, Matsuda S, Hikishima M, Oyama S, Ogawa Y, Kurita S, Fujii R (2018) Electrostatic electron cyclotron harmonic waves as a candidate to cause pulsating auroras. Geophys Res Lett 45:12661-12668. https:// doi.org/10.1029/2018GL080145

Hanaoka Y, Katsukawa Y, Morita S, Kamata Y, Ishizuka N (2020) A HAWAll2RG infrared camera operated under fast readout mode for solar polarimetry. Earth Planets Space 72:181. https://doi.org/10.1186/ s40623-020-01318-8

Hanaoka Y, Sakurai T (2017) Statistical study of the magnetic field orientation in solar filaments. Astrophys J 851:130. https://doi.org/10.3847/15384357/aa9cf1

Hayakawa H, Clette F, Horaguchi T, Iju T, Knipp DJ, Liu H, Nakajima T (2020a) Sunspot Observations by Hisako Koyama: 1945-1996. Mon Not R Astron Soc 492(3):4513-4527. https://doi.org/10.1093/mnras/stz3345

Hayakawa H, Besser BP, Iju T, Arlt R, Uneme S, Imada S, Bourdin P-A, Kraml A (2020b) Thaddäus Derfflinger's sunspot observations during 1802-1824: a primary reference to understand the Dalton Minimum. Astrophys J 890:98. https://doi.org/10.3847/1538-4357/ab65c9

Hayakawa H, Owens MJ, Lockwood M, Soma M (2020c) The solar corona during the total eclipse on 16 June 1806: Graphical evidence of the corona structure during the Dalton Minimum. Astrophys J 900:114. https://doi. org/10.3847/1538-4357/ab9807

Hinode Review Team, Al-Janabi K, Antolin P, Baker D, Bellot Rubio LR, Bradley L, Brooks DH, Centeno R, Culhane JL, Del Zanna G, Doschek GA, Fletcher L, Hara H, Harra LK, Hillier AS, Imada S, Klimchuk JA, Mariska JT, Pereira TMD, Reeves KK, Sakao T, Sakurai T, Shimizu T, Shimojo M, Shiota D, Solanki SK, Sterling AC, Su Y, Suematsu Y, Tarbell TD, Tiwari SK, Toriumi S, Ugarte-Urra I, Warren HP, Watanabe T, Young PR (2019) Achievements of Hinode in the first eleven years. PASJ 71:R1. https://doi.org/10.1093/ pasj/psz084

Hirai A, Tsuchiya F, Obara T, Kasaba Y, Katoh Y, Misawa H, Shiokawa K, Miyoshi Y, Kurita S, Matsuda S, Connors M, Nagatsuma T, Sakaguchi K, Kasahara Y, Kumamoto A, Matsuoka A, Shoji M, Shionohara I, Albert JM (2018) Temporal and spatial correspondence of PC1/EMIC waves and relativistic electron precipitations observed with ground-based multi-instruments on 27 March 2017. Geophys Res Lett 45:24. https://doi.org/10.1029/ 2018GL080126

Hosoda S, Muranaka T, Kuninaka H, Kim J, Hatta S, Kurahara N, Cho M, Ueda HO, Koga K, Goka T (2008) Laboratory experiments for code validation 
of multiutility spacecraft charging analysis tool (MUSCAT). IEEE Trans Plasma Sci 36(5):2350-2359. https://doi.org/10.1109/TPS.2008.2003973

Hosokawa K, Sakai J, Tomizawa I, Saito S, Tsugawa T, Nishioka M, Ishii M (2020) A monitoring network for anomalous propagation of aeronautical VHF radio waves due to sporadic E in Japan, Earth. Planets Space 72:88. https://doi.org/10.1186/s40623-020-01216-z

Hotta H, lijima H, Kusano K (2019) Weak influence of near-surface layer on solar deep convection zone revealed by comprehensive simulation from base to surface. Sci Adv 5(1):2307. https://doi.org/10.1126/sciadv.aau23 07

Hotta H, Rempel M, Yokoyama T (2016) Large-scale magnetic fields at high Reynolds numbers in magnetohydrodynamic simulations. Science 351(6280):1427-1430. https://doi.org/10.1126/science.aad1893

Hozumi K, Kondo T, Saito S, Nakata H, Maruyama T, Tsugawa T, Ishii M (2018) Low cost development of HF receiver prototype for HF-START field campaign, In: Proceeding of 2nd URSI Atlantic Radio Science Meeting (AT-RASC), Meloneras, Spain, pp. 1-4. https://doi.org/10.23919/URSI-ATRASC.2018.8471477

Hozumi K, Ishii M, Saito S, Maruyama T, Nakata H, Tsugawa T (2019) HF-START: application in aid of radio communications/navigation, In: Electronic Navigation Research Institute (eds) Air Traffic Management and Systems III. EIWAC 2017. Lecture Notes in Electrical Engineering. vol. 555. Springer, Singapore. https://doi.org/10.1007/978-981-13-7086-1_19

Ichimoto K, Ishii TT, Otsuji K, Kimura G, Nakatani Y, Kaneda N, Nagata S, Ueno S, Hirose K, Cabezas D, Morita S (2017) A new solar imaging system for observing high speed eruptions: solar dynamics Doppler imager (SDDI). Sol Phys 292:63. https://doi.org/10.1007/s11207-017-1082-7

lijima H, Hotta H, Imada S (2019) Effect of morphological asymmetry between leading and following sunspots on the prediction of solar cycle activity. Astrophys J 883(1):24. https://doi.org/10.3847/1538-4357/ab3b04,1908. 04474

lijima H, Hotta H, Imada S, Kusano K, Shiota D (2017) Improvement of solarcycle prediction: plateau of solar axial dipole moment. Astron Astrophys 607:L2. https://doi.org/10.1051/0004-6361/201731813

Imada S, Fujiyama M (2018) Effect of magnetic field strength on solar differential rotation and meridional circulation. Astrophys J Letter 864(1):L5. https://doi.org/10.3847/2041-8213/aad904

Imada S, Matobe K, Fujiyama M, lijima H (2020) Solar cycle related variation in solar differential rotation and meridional flow in Cycle 24. Earth Planets Space 72:182. https://doi.org/10.1186/s40623-020-01314-y

Ingham M (1993) Analysis of variations in cathodic protection potential and corrosion risk on the natural gas pipeline at Dannevirke. Report for the Natural Gas Corporation, 94

Inoue S, Kusano K, Büchner J, Skála J (2018a) Formation and dynamics of a solar eruptive flux tube. Nat Commun 9:174. https://doi.org/10.1038/ s41467-017-02616-8

Inoue S, Shiota D, Bamba Y, Park S-H (2018b) Magnetohydrodynamic modeling of a solar eruption associated with an X9.3 flare observed in the Active Region 12673. Astrophys J 867:83. https://doi.org/10.3847/1538-4357/ aae079

Ishiguro N, Kusano K (2017) Double arc instability in the solar corona. Astrophys J 843:101. https://doi.org/10.3847/1538-4357/aa799b

Ishii M, Shiota D, Tao C, Ebihara Y, Fujiwara H, Ishii T, Ichimoto K, Kataoka R, Koga K, Kubo Y, Kusano K, Miyoshi Y, Nagatsuma T, Nakamizo A, Nakamura M, Nishioka M, Saito S, Sato T, Tsugawa T, Yoden S (2021) Space weather benchmarks on Japanese society. Earth Planets Space 73:108. https://doi.org/10.1186/s40623-021-01420-5

Iwai K, Kubo Y, Ishibashi H, Naoi T, Harada K, Ema K, Hayashi Y, Chikahiro Y (2017) OCTAD-S: digital fast Fourier transform spectrometers by FPGA. Earth Planets Space 69:95. https://doi.org/10.1186/s40623-017-0681-8

I wai K, Shiota D, Tokumaru M, Fujiki K, Den M, Kubo Y (2019) Development of a coronal mass ejection arrival time forecasting system using interplanetary scintillation observations. Earth Planets Space 71:39. https://doi. org/10.1186/s40623-019-1019-5

I wai K, Shiota D, Tokumaru M, Fujiki K, Den M, Kubo Y (2021) Validation of coronal mass ejection arrival-time forecasts by magnetohydrodynamic simulations based on interplanetary scintillation observations. Earth Planets Space 73:9. https://doi.org/10.1186/s40623-020-01345-5

I wai K, Yashiro S, Nitta NV, Kubo Y (2020) Spectral structures of type II solar radio bursts and solar energetic particles. Astrophys J 888:50. https:// doi.org/10.3847/1538-4357/ab57ff
Jin H, Miyoshi Y, Fujiwara H, Shinagawa H, Terada K, Terada N, Ishii M, Otsuka Y, Saito A (2011) Vertical connection from the tropospheric activities to the ionospheric longitudinal structure simulated by a new Earth's whole atmosphere-ionosphere coupled model. J Geophys Res 116:A01316. https://doi.org/10.1029/2010JA015925

Jin H, Miyoshi Y, Pancheva D, Mukhtarov P, Fujiwara H, Shinagawa H (2012) Response of migrating tides to the stratospheric sudden warming in 2009 and their effects on the ionosphere studied by a whole atmosphere-ionosphere model GAIA with COSMIC and TIMED/SABER observations. J Geophys Res 117:A10323. https://doi.org/10.1029/2012J A017650

Kakuwa J, Ueno S (2021) Investigation of the long-term variation of solar call K intensity. I. Density-to-intensity calibration formula for historical photographic plates. Astrophys J Suppl Ser 254:44. https://doi.org/10. 3847/1538-4365/abfbe3

Kasahara S, Miyoshi Y, Yokota S, Mitani T, Kasahara Y, Matsuda S, Kumamoto A, Matsu A, Kazama Y, Frey HU, Anvelopoulos V, Kurita S, Keika K, Seki K, Shinohara I (2018) Pulsating aurora from electron scattering by chorus waves. Nature 554:337-340. https://doi.org/10.1038/nature25505

Kataoka R, Sato T, Miyake S, Shiota D, Kubo Y (2018) Radiation dose nowcast for the ground level enhancement on 10-11 September 2017. Space Weather 16:917-923. https://doi.org/10.1029/2018SW001874

Katoh Y, Omura Y (2016) Electron hybrid code simulation of whistler-mode chorus generation with real parameters in the Earth's inner magnetosphere. Earth Planets Space 68:192. https://doi.org/10.1186/ s40623-016-0568-0

Katoh Y, Omura Y, Miyake Y, Usui H, Nakashima H (2018) Dependence of generation of whistler mode chorus emissions on the temperature anisotropy and density of energetic electrons in the Earth's inner magnetosphere. J Geophys Res Space Physics 123:1165-1177. https://doi. org/10.1002/2017JA024801

Kaufman AA, Keller GV (1981) The magnetotelluric sounding method. Method Geochem Geophys 15

Kawabata Y, lida Y, Doi T, Akiyama S, Yasiro S, Shimizu T (2018) Statistical relation between solar flares and coronal mass ejections with respect to sigmoidal structures in active regions. Astrophys J 869:99. https://doi. org/10.3847/1538-4357/aaebfc

Kawabata Y, Inoue S, Shimizu T (2020) Extrapolation of three-dimensional magnetic field structure in flare-productive active regions with different initial conditions. Astrophys J 895:105. https://doi.org/10.3847/15384357/ab8ea9

Kawai T, Imada S, Nishimoto S, Watanabe K, Kawate T (2020) Nowcast of an EUV dynamic spectrum during solar flares. JASTP 205:105302. https:// doi.org/10.1016/j.jastp.2020.105302

Kihara K, Huang Y, Nishimura N, Nitta NV, Yashiro S, Ichimoto K, Asai A (2020) Statistical analysis of the relation between coronal mass ejections and solar energetic particles. Astrophys J 900:75. https://doi.org/10.3847/ 1538-4357/aba621

Kitai R, Kadota M, Shirakawa S, Hada Y (2014) Digital archiving of solar synoptic observation (1) Project outline and meta-data archiving. Technical Reports from Kwasan and Hida Observatories, Graduate School of Science, Kyoto University 2-2:8

Kitamura N, Kitahara M, Shoji M, Miyoshi Y, Hasegawa H, Nakamura S, Katoh Y, Saito Y, Yokota S, Gershman DJ, Vinas AF, Giles BL, Moore TE, Paterson WR, Pollock CJ, Russell CT, Strangeway RJ, Fuselier SA, Burch JL (2018) Direct measurements of two-way wave-particle energy transfer in a collisionless space plasma. Science 361:1000-1003. https://doi.org/10. 1126/science.aap8730

Koen J, Gaunt T (2003) Geomagnetically induced currents in the Southern African electricity transmission network, IEEE Bologna Power Tech Conference, 23-26 June 2003, Bologna, Italy. https://doi.org/10.1109/ PTC.2003.1304165

Kubo Y, Kataoka R, Sato T (2015) Interplanetary particle transport simulation for warning system for aviation exposure to solar energetic particles. Earth Planets Space 67:117. https://doi.org/10.1186/s40623-015-0260-9

Kubota Y, Nagatsuma T, Nakamizo A, Sakaguchi K, Den M, Matsumoto H, Higashio N, Tanaka T (2019) Comparison of magnetospheric magnetic field variations at quasi-zenith orbit based on Michibiki observation and REPPU global MHD simulation. IEEE Trans Plasma Sci 47:8. https://doi. org/10.1109/TPS.2019.2910301 
Kurita S, Miyoshi Y, Kasahara S, Yokota S, Kasahara Y, Matsuda S, Kumamoto A, Matsuoka A, Shinohara I (2018) Deformation of electron pitch angle distributions caused by upper-band chorus observed by the Arase satellite. Geophys Res Lett 45:7996-8004. https://doi.org/10.1029/ 2018GL079104

Kurita S, Miyoshi Y, Shiokawa K, Higashio N, Mitani T, Takashima T et al (2018b) Rapid loss of relativistic electrons by EMIC waves in the outer radiation belt observed by Arase, Van Allen Probes, and the PWING ground stations. Geophys Res Lett 45:12720-12729. https://doi.org/10.1029/ 2018GL080262

Kusano K, lju T, Bamba Y, Inoue S (2020) A physics-based method that can predict imminent large solar flares. Science 369:587-591. https://doi. org/10.1126/science.aaz2511

Leka KD, Park S-H, Kusano K, Andries J, Barnes G, Bingham S et al (2019a) A comparison of flare forecasting methods. Il. Benchmarks, metrics, and performance results for operational solar flare forecasting systems. Astrophys J Suppl Ser 243:36. https://doi.org/10.3847/1538-4365/ ab2e12

Leka KD, Park S-H, Kusano K, Andries J, Barnes G, Bingham S et al (2019b) A comparison of flare forecasting methods. III. Systematic behaviors of operational solar flare forecasting systems. Astrophys J 881:101. https:// doi.org/10.3847/1538-4357/ab2e11

Lin PH, Kusano K, Leka KD (2021) Eruptivity in solar flares: the challenges of magnetic flux ropes. Astrophys J 913:124. https://doi.org/10.3847/15384357/abf3c1

Lin PH, Kusano K, Shiota D, Inoue S, Leka KD, Mizuno Y (2020) A new parameter of the photospheric magnetic field to distinguish eruptive-flare producing solar active regions. Astrophys J 894:20. https://doi.org/10.3847/ $1538-4357 / a b 822 c$

Liu C, Liu L, Yang Y (2009) Monitoring and modeling geomagnetically induced currents in power grids of China. Paper presented at 2009 Asia-Pacific Power and Engineering Conference, Wuhan, China, 27-31 March 2009, https://doi.org/10.1109/APPEEC.2009.4918502.

Mackie RL, Smith JT, Madden TR (1994) Three-dimensional electromagnetic modeling using finite difference equations: the magnetotelluric example. Radio Sci 29(4):923-935. https://doi.org/10.1029/94rs00326

Marshall RA et al (2012) Geomagnetically induced currents in the New Zealand power network. Space Weather 10(8):S08003. https://doi.org/10. 1029/2012SW000806

Marshall RA, Gorniak H, Walt TVD, Waters CL, Sciffer MD, Miller M, Dalzell M, Daly T, Pouferis G, Hesse G, Wilkinson P (2013) Observations of geomagnetically induced currents in the Australian power network. Space Weather 11(1):6-16. https://doi.org/10.1029/2012SW000849

Miyahara H, Higuchi C, Terasawa T, Kataoka R, Sato M, Takahashi Y (2017a) Solar 27-day rotational period detected in a wide-area lightning activity in Japan. ANGEO Commun 35:583-588. https://doi.org/10.5194/ angeo-35-583-2017

Miyahara H, Aono Y, Kataoka R (2017b) Searching for the 27-day solar rotational cycle in lightning events recorded in old diaries in Kyoto from the 17th to 18th century. ANGEO Commun 35:1 195-1200. https://doi.org/ 10.5194/angeo-35-1195-2017

Miyahara H, Kataoka R, Mikami T, Zaiki M, Hirano J, Yoshimura M, Aono Y, Iwahashi K (2018) Solar rotational cycle in lightning activity in Japan during the 18-19th centuries. ANGEO Commun 36:633-640. https://doi.org/ 10.5194/angeo-36-633-2018

Miyahara H, Horiuchi K, Sakashita W, Matsuzaki H, Zhou L, Xu H (2020) Measurement of beryllium-10 in terrestrial carbonate deposits from South China: a pilot study. Nucl Instrum Methods Phys Res, Sect B 464:36-40. https://doi.org/10.1016/..nimb.2019.11.036

Miyake F, Nagaya K, Masuda K, Nakamura T (2012) A signature of cosmic-ray increase in AD 774-775 from tree rings in Japan. Nature 486:240-242. https://doi.org/10.1038/nature11123

Miyoshi T, Kusano K, Inoue S (2020) A magnetohydrodynamic relaxation method for non-force-free magnetic field in magnetohydrostatic equilibrium. Astrophys J Suppl Ser 247:6. https://doi.org/10.3847/15384365/ab64f2

Miyoshi Y, Shinohara I, Takashima T, Asamura K, Higashio N, Mitani T, Kasahara S, Yokota S, Kazama Y, Wang S-Y, Tam SW, Ho PTP, Kasahara Y, Kasaba Y, Yagitani S, Matsuoka A, Kojima H, Katoh H, Shiokawa K, Seki K (2018) Geospace exploration project ERG. Earth Planets Space 70:101. https:// doi.org/10.1186/s40623-018-0862-0
Miyoshi YS, Jordanova VK, Morioka A, Evans DS (2004) Solar cycle variations of the electron radiation belts: observations and radial diffusion simulation. Space Weather 2:S10S02. https://doi.org/10.1029/2004SW000070

Miyoshi Y, Kataoka R, Ebihara Y (2016) Flux enhancement of relativistic electrons associated with substorms. In: Balasis G, Daglis IA, Mann IR (eds) Waves, particles, and storms in geospace. Oxford Press, pp. 333-353

Moral AC, Shiokawa K, Suzuki S, Liu H, Otsuka Y, Yatini CY (2019) Observations of low-latitude traveling ionospheric disturbances by a 630.0-nm airglow imager and the CHAMP satellite over Indonesia. J Geohphys Res 124:2198-2212. https://doi.org/10.1029/2018JA025634

Moriya T, Miyahara H, Ohyama M, Hakozaki M, Takeyama M, Sakurai H, Tokanai F (2019) A study of variation of the 11-year solar cycle before the onset of the Spoerer Minimum based on annually measured $14 \mathrm{C}$ content in tree rings. Radiocarbon 61:1749-1754. https://doi.org/10.1017/RDC. 2019.123

Muranaka T, Hosoda S, Kim JH, Hatta S, Ikeda K, Hamanaga T, Cho M, Usui H, Ueda HO, Koga K, Goka T (2008) Development of multi-utility spacecraft charging analysis tool (MUSCAT). IEEE Trans Plasma Sci 36(5):2336-2349. https://doi.org/10.1109/TPS.2008.2003974

NASA-HDBK-4002A (2017) Mitigating in-space charging effects—a guideline, NASA Technical Handbook

Nagatsuma T, Nakamizo A, Kubota Y, Nakamura M, Koga K, Miyoshi Y, Matsumoto H (2021) Development of space environment customized risk estimation for satellites (SECURES). Earth Planets Space 73:26. https:// doi.org/10.1186/s40623-021-01355-x

Nakajima H, Murata I, Nagahama Y, Akiyoshi H, Saeki K, Kinase T, Takeda M, Tomikawa Y, Dupuy E, Jones NB (2020) Chlorine partitioning near the polar vortex edge observed with ground-based FTIR and satellites at Syowa Station, Antarctica, in 2007 and 2011. Atmos Chem Phys 20:1043-1074. https://doi.org/10.5194/acp-20-1043-2020

Nakamura S, Ebihara Y, Fujita S, Goto T, Yamada N, Watari S, Omura Y (2018) Time domain simulation of geomagnetically induced current (GIC) flowing in $500 \mathrm{kV}$ power grid in Japan including a three-dimensional ground inhomogeneity. Space Weather 16:1946-1959. https://doi.org/ 10.1029/2018SW002004

Nakamura S, Omura Y, Kletzing C, Baker DN (2019) Rapid precipitation of relativistic electron by EMIC rising-tone emissions observed by the Van Allen Probes. J Geophys Res Space Physics 124:6701-6714. https://doi. org/10.1029/2019JA026772

Nakamura Y, Shiokawa K, Otsuka Y, Oyama S-I, Nozawa S, Komolmis T, Komonjida S, Neudegg D, Yuile C, Meriwether J, Shinagawa H, Jin H (2017) Measurement of thermospheric temperatures using OMTI Fabry-Perot interferometers with $70 \mathrm{~mm}$ etalon. Earth Planets Space 69:57. https:// doi.org/10.1186/s40623-017-0643-1

Naoe H, Yoshida K (2019) Influence of quasi-biennial oscillation on the boreal winter extratropical stratosphere in QBOi experiments. Q J R Meteorol Soc 145:2755-2771. https://doi.org/10.1002/qj.3591

National Research Council (2008) Severe space weather events: Understanding societal and economic impacts: a workshop report, Washington, D.C., The National Academies Press. https://doi.org/10.17226/12507

Nishimoto S, Watanabe K, Imada S, Kawate T, Lee K-S (2020) Statistical and observational research on solar flare EUV spectra and geometrical features. Astrophys J 904:31. https://doi.org/10.3847/1538-4357/abbacb

Nishimoto S, Watanabe K, Kawai T, Imada S, Kawate T (2021) Validation of computed extreme ultraviolet emission spectra during solar flares. Earth Planets Space 73:79. https://doi.org/10.1186/s40623-021-01402-7

Nishioka M, Tsugawa T, Jin H, Ishii M (2017) A new ionospheric storm scale based on TEC and foF2 statistics. Space Weather 15:228-239. https:// doi.org/10.1002/2016SW001536

Nishizuka N, Kubo Y, Sugiura K, Den M, Ishii M (2020) Reliable probability forecast of solar flares: deep flare net-reliable (DeFN-R). Astrophys J 899:150. https://doi.org/10.3847/1538-4357/aba2f2

Nishizuka N, Kubo Y, Sugiura K, Den M, Ishii M (2021) Operational solar flare prediction model using deep flare net. Earth Planets Space 73:64. https://doi.org/10.1186/s40623-021-01381-9

Nishizuka N, Sugiura K, Kubo Y, Den M, Ishii M (2018) Deep Flare Net (DeFN) model for solar flare prediction. Astrophys J 858:113. https://doi.org/10. 3847/1538-4357/aab9a7

Noda S, Kodera K, Adachi Y, Deushi M, Kitoh A, Mizuta R, Murakami S, Yoshida K, Yoden S (2017) Impact of interactive chemistry of stratospheric 
ozone on Southern Hemisphere paleoclimate simulation. J Geophys Res 122:878. https://doi.org/10.1002/2016JD025508

Noda S, Kodera K, Adachi Y, Deushi M, Kitoh A, Mizuta R, Murakami S, Yoshida K, Yoden S (2018) Mitigation of global cooling by stratospheric chemistry feedbacks in a simulation of the Last Glacial Maximum. J Geophys Res 123:9378. https://doi.org/10.1029/2017JD028017

Oka M, Obara T, Nitta NV, Yashiro S, Shiota D, Ichimoto K (2021) Unusual enhancement of $\sim 30 \mathrm{MeV}$ proton flux in an ICME sheath region. Earth, Planets Space 73:31. https://doi.org/10.1186/s40623-021-01362-y

Otsuka Y, Onoma F, Shiokawa K, Ogawa T, Yamamoto M, Fukao S (2007) Simultaneous observations of nighttime medium-scale traveling ionospheric disturbances and E region field-aligned irregularities at midlatitude. J Geophys Res 112:A06317. https://doi.org/10.1029/2005JA011548

Otsuka Y, Tani T, Tsugawa T, Ogawa T, Saito A (2008) Statistical study of relationship between medium-scale traveling ionospheric disturbance and sporadic E layer activities in summer night over Japan. J Atmos SolarTerr Phys 70:2196-2202. https://doi.org/10.1016/j.jastp.2008.07.008

Park S-H, Leka KD, Kusano K, Andries J, Barnes G, Bingham S et al (2020) A comparison of flare forecasting methods. IV. Evaluating consecutiveday forecasting patterns. Astrophys J 890:124. https://doi.org/10. 3847/1538-4357/ab65fo

Pesnell WD (2016) Predictions of solar cycle 24: how are we doing? Space Weather 14:10-21. https://doi.org/10.1002/20155W001304

Pirjola R (1985) On currents induced in power transmission systems during geomagnetic variations. IEEE Power Eng Rev PER 5(10):42-43. https:// doi.org/10.1109/MPER.1985.5528

Pulkkinen A, Pirjola R, Viljanen A (2007) Determination of ground conductivity and system parameters for optimal modeling of geomagnetically induced current flow in technological systems. Earth Planets Space 59(9):999-1006. https://doi.org/10.1186/BF03352040

Rodi WL (1976) A technique for improving the accuracy of finite element solutions for magnetotelluric data. Geophys J Int 44(2):483-506. https://doi.org/10.1111/j.1365-246X.1976.tb03669.x

Roussel JF, Rogier F, Dufour G, Mateo-Velez JC, Forest J, Hilgers A, Rodgers D, Girard L, Payan D (2008) SPIS open-source code: methods, capabilities, achievements, and prospects. IEEE Trans Plasma Sci 36(5):23602368. https://doi.org/10.1109/TPS.2008.2002327

Saito S, Miyoshi Y, Seki K (2016) Rapid increase in relativistic electron flux controlled by nonlinear phase trapping of whistler chorus elements. J Geophys Res Space Physics 121:6573-6589. https://doi.org/10. 1002/2016JA022696

Saito S, Suzuki S, Yamamoto M, Saito A, Chen C (2017) Real-time ionosphere monitoring by three-dimensional tomography over Japan. Navigation 64:495-504. https://doi.org/10.1002/navi.213

Saito S, Yamamoto M, Hashiguchi H, Maegata A, Saito A (2007) Observational evidence of coupling between quasi-periodic echoes and medium scale traveling ionospheric disturbances. Ann Geophys 25:2185-2194. https://doi.org/10.5194/angeo-25-2185-2007

Sakai J, Hosokawa K, Tomizawa I, Saito S (2019) A statistical study of anomalous VHF propagation due to the sporadic-E layer in the air-navigation band. Radio Sci 54:426-439. https://doi.org/10.1029/ 2018RS006781

Sakai J, Saito S, Hosokawa K, Tomizawa I (2020) Anomalous propagation of radio waves from distant ILS localizers due to ionospheric sporadic-E. Space Weather 18:e2020SW002517. https://doi.org/10.1029/2020S W002517

Sakashita W, Miyahara H, Yokoyama Y, Aze T, Nakatsuka T, Hoshino Y, Ohyama M, Yonenobu H, Takemura K (2017) Hydroclimate reconstruction in central Japan over the past four centuries from tree-ring cellulose 8180. Quatern Int 455:1-7. https://doi.org/10.1016/j.quaint.2017.06. 020

Sakharov YaA, Danilin AN, Ostafiychuk RM, Katkalov YuV, Kudryashova NV (2009) Geomagnetically induced currents in the power systems of the Kola Peninsula at solar minimum. In: Proceedings of 8 th international symposium on electromagnetic compatibility and electromagnetic ecology, St. Petersburg, pp. 237-238

Sakurai T, Hanaoka Y, Arai T, Hagino M, Kawate T, Kitagawa N, Kobiki T, Miyashita M, Morita S, Otsuji K, Shinoda K, Suzuki I, Yaji K, Yamasaki T, Fukuda T, Noguchi M, Takeyama N, Kanai Y, Yamamuro T (2018) Infrared spectropolarimeter on the solar flare telescope at NAOJ/Mitaka. PASJ 70:58. https://doi.org/10.1093/pasj/psy050
Sato T, Kataoka R, Shiota D, Kubo Y, Ishii M, Yasuda H, Miyake S, Park IC, Miyoshi Y (2018a) Real time and automatic analysis program for WASAVIES: warning system for aviation exposure to solar energetic particles. Space Weather 16:924-936. https://doi.org/10.1029/2018SW001873

Sato T, Iwamoto Y, Hashimoto S, Ogawa T, Furuta T, Abe S, Kai T, Tsai PE, Matsuda N, Iwase H, Shigyo N, Sihver L, Niita K (2018b) Features of particle and heavy ion transport code system PHITS Version 3.02. J Nucl Sci Technol 55:684-690. https://doi.org/10.1080/00223131.2017.1419890

Sato T, Kataoka R, Shiota D, Kubo Y, Ishii M, Yasuda H, Miyake S, Miyoshi Y, Ueno $\mathrm{H}$, Nagamatsu A (2019) Nowcast and forecast of galactic cosmic ray (GCR) and solar energetic particle (SEP) fluxes in magnetosphere and ionosphere-extension of WASAVIES to Earth orbit. J Space Weather Space Clim 9:A9. https://doi.org/10.1051/swsc/2019006

Sato T, Kataoka R, Yasuda H, Yashiro S, Kuwabara T, Shiota D, Kubo Y (2014) Air shower simulation for WASAVIES: warning system for aviation exposure to solar energetic particles. Radiat Prot Dosim 161:274-278. https://doi. org/10.1093/rpd/nct332

Seki D, Otsuji K, Ishii TT, Asai A, Ichimoto K (2021) Relationship between threedimensional velocity of filament eruptions and CME association. Earth Planets Space 73:58. https://doi.org/10.1186/s40623-021-01378-4

Seki D, Otsuji K, Isobe H, Ishii TT, Ichimoto K, Shibata K (2019) Small-scale motions in the solar filaments as the precursors of the eruptions. PASJ 71:56. https://doi.org/10.1093/pasj/psz031

Seki D, Otsuji K, Isobe H, Ishii TT, Sakaue T, Hirose K (2017) Increase in the amplitude of line-of-sight velocities of the small-scale motions in a solar filament before eruption. Astrophys J 843:L24. https://doi.org/10. 3847/2041-8213/aa7559

Shimojo M, Iwai K, Asai A, Nozawa S, Minamidani T, Saito M (2017) Variation of the solar microwave spectrum in the last half century. Astrophys J 848:62. https://doi.org/10.3847/1538-4357/aa8c75

Shinagawa H, Jin H, Miyoshi Y, Fujiwara H, Yokoyama T, Otsuka Y (2018) Daily and seasonal variations in the linear growth rate of the Rayleigh-Taylor instability in the ionosphere obtained with GAIA. Prog Earth Planet Sci 5:16. https://doi.org/10.1186/s40645-018-0175-8

Shinagawa H, Miyoshi Y, Jin H, Fujiwara H (2017) Global distribution of neutral wind shear associated with sporadic $E$ layers derived from GAIA. J Geophys Res Space Physics 122:4450-4465. https://doi.org/10.1002/ 2016JA023778

Shiota D, Kataoka R (2016) Magnetohydrodynamic simulation of interplanetary propagation of multiple coronal mass ejections with internal magnetic flux rope (SUSANOO-CME). Space Weather 14:56-75. https://doi.org/10. 1002/2015SW001308

Shiota D (2020) Real-time prediction of solar storm impact. NICT news 479:8. Available via http://www.nict.go.jp/en/data/nict-news/NICT_NEWS_ 2020-479_E.pdf. Accessed 2 Dec 2020

Shoji M, Miyoshi Y, Katoh Y, Keika K, Angelopoulos V, Kasahara S, Asamura K, Nakamura S, Omura Y (2017) Ion hole formation and nonlinear generation of electromagnetic ion cyclotron waves: THEMIS observations. Geophys Res Lett 44:8730-8738. https://doi.org/10.1023/2017GL0742 54

Taguchi S, Shibata H (1961) World map of foEs. J Radio Res Lab 8:355-389

Takeo D, Shiokawa K, Fujinami H, Otsuka Y, Matsuda TS, Ejiri MK, Nakamura T, Yamamoto M (2017) Sixteen-year variation of horizontal phase velocity and propagation direction of mesospheric and thermospheric waves in airglow images at Shigaraki, Japan. J Geophys Res 122:8770-8780. https://doi.org/10.1002/2017JA023919

Tanaka T, Ebihara Y, Watanabe M, Den M, Fujita S, Kikuchi T, Hashimoto KK, Kataoka R (2019) Development of magnetic topology during the growth phase of the substorm inducing the onset of the near-Earth neutral line. J Geophys Res Space Physics 124:5158. https://doi.org/10. 1029/2018JA026386

Tanaka T (2015) Substorm auroral dynamics reproduced by advanced global magnetosphere-ionosphere (M-I) coupling simulation. In: Zhang Y (ed) Auroral dynamics and space weather. Geophys Monogr Ser. Vol. 215 AGU, Washington DC. p. 177. https://doi.org/10.1002/9781118978719. ch13

Tao C, Jin H, Miyoshi Y, Shinagawa H, Fujiwara H, Nishioka M, Ishii M (2020) Numerical forecast of the upper atmosphere and ionosphere using GAIA. Earth Planets Space 72:178. https://doi.org/10.1186/ s40623-020-01307-x 
Teramoto M, Hori T, Saito S, Miyoshi Y, Kurita S, Higashio N, Matsuoka A, Kasahara Y, Kasaba Y, Takashima T, Nomura R, Nosé M, Fujimoto A, Tanaka Y-M, Shoji M, Tsugawa Y, Shinohara M, Shinohara I, Blake JB, Fennell JF, Claudepierre SG, Turner DL, Kletzing CA, Sormakov D, Troshichev O (2019) Remote detection of drift resonance between energetic electrons and ultralow frequency waves: multisatellite coordinated observation by Arase and Van Allen Probes. Geophys Res Lett 46:11642-11651. https://doi.org/10.1029/2019GL084379

Toriumi S, Hotta H (2019) Spontaneous generation of $\delta$-sunspots in convective magnetohydrodynamic simulation of magnetic flux emergence. Astrophys J Lett 886:21. https://doi.org/10.3847/2041-8213/ab55e7

Toriumi S, Schrijver CJ, Harra LK, Hudson H, Nagashima K (2017) Magnetic properties of solar active regions that govern large solar flares and eruptions. Astrophys J 834:56. https://doi.org/10.3847/1538-4357/ $834 / 1 / 56$

Toriumi S, Takasao S (2017) Numerical simulations of flare-productive active regions: $\delta$-sunspots, sheared polarity inversion lines, energy storage, and predictions. Astrophys J 850:39. https://doi.org/10.3847/15384357/aa95c

Toriumi S, Wang H (2019) Flare-productive active regions. Living Rev Sol Phys 16:3. https://doi.org/10.1007/s41116-019-0019-7

Torta JM, Marsal S, Quintana M (2014) Assessing the hazard from geomagnetically induced currents to the entire high-voltage powe network in Spain. Earth Planet Space 66:87. https://doi.org/10.1186/ 1880-5981-66-87

Tsuchiya S, Shiokawa K, Fujinami H, Otsuka Y, Nakamura T, Connors M, Schofield I, Shevtsov B, Poddelsky I (2019) Three-dimensional Fourier analysis of the phase velocity distributions of mesospheric and ionospheric waves based on airglow images collected over 10 years: comparison of Magadan, Russia, and Athabasca, Canada. J Geophys Res 124:8110 8124. https://doi.org/10.1029/2019JA026783

Tsuchiya S, Shiokawa K, Fujinami H, Otsuka Y, Nakamura T, Yamamoto M (2018) Statistical analysis of the phase velocity distribution of mesospheric and ionospheric waves observed in airglow images over a 16-year period: comparison between Rikubetsu and Shigaraki, Japan. J Geophys Res 123:6930-6947. https://doi.org/10.1029/2018JA025585

Tsuchiya S, Shiokawa K, Otsuka Y, Nakamura T, Yamamoto M, Connors M, Schofield I, Schevtsov B, Poddelsky I (2020) Wavenumber spectra of atmospheric gravity waves and medium-scale traveling ionospheric disturbances based on more than 10-year airglow images in Japan, Russia, and Canada. J Geophys Res 125:e26807. https://doi.org/10.1029/ 2019JA026807

Viljanen A, Pirjola R (1994) Geomagnetically induced currents in the Finnish high-voltage power system. Surv Geophys 15(4):383-408. https://doi. org/10.1007/BF00665999
Watanabe $\mathrm{K}$, Jin $\mathrm{H}$, Nishimoto $\mathrm{S}$, Imada S, Kawai T, Kawate T, Otsuka Y Shinbori A, Tsugawa T, Nishioka M (2021) Model-based reproduction and validation of the total spectrum of solar flare and their impact on the global environment at the X93 event of September 62017 . Earth Planets Space 73:96. https://doi.org/10.1186/s40623-021-01376-6

Watari S, Kunitake M, Kitamura K, Hori T, Kikuchi T, Shiokawa K, Nishitani N, Kataoka R, Kamide Y, Aso T, Watanabe Y, Tsuneta Y (2009) Measurements of geomagnetically induced current in a power grid in Hokkaido, Japan. Space Weather 7:S03002. https://doi.org/10.1029/2008SW000417

Watari S, Nakamura S, Ebihara Y (2021) Measurement of geomagnetically induced current (GIC) around Tokyo, Japan. Earth Planets Space 73:102. https://doi.org/10.1186/s40623-021-01422-3

Wik M et al (2009) Space weather events in July 1982 and October 2003 and the effects of geo-magnetically induced currents on Swedish technical systems. Ann Geophys 27:1775-1787. https://doi.org/10.5194/ angeo-27-1775-2009

Xu H, Miyahara H, Horiuchi K, Matsuzaki H, Sun H, Luo W, Zheng X, Suganuma Y, Wang S, Zhou L (2019) High-resolution records of 10Be in endogenic travertine from Baishuitai, China: a new proxy record of annual solar activity? Quatern Sci Rev 216:34-46. https://doi.org/10.1016/j.quascirev. 2019.05.012

Yokoyama T, Jin H, Shinagawa H, Liu H (2019) Seeding of equatorial plasma bubbles by vertical neutral wind. Geophys Res Lett 46:7088-7095. https://doi.org/10.1029/2019GL083629

Yoshikawa A, Fujii R (2018) Earth's ionosphere: theory and phenomenology of cowling channels. In: Keiling A, Marghitu O, Wheatland M (eds) Electric currents in geospace and beyond (AGU-Book). Wiley, Hoboken. https:// doi.org/10.1002/9781119324522.ch25

Yukimoto S, Adachi Y, Hosaka M, Sakami T, Yoshimura H, Hirabara M, Tanaka TY, Shindo E, Tsujino H, Deushi M, Mizuta R, Yabu S, Obata A, Nakano H, Koshiro T, Ose T, Kitoh A (2012) A new global climate model of the Meteorological Research Institute: MRI-CGCM3-Model description and basic performance-. J Meteor Soc Japan 90A:23-64. https://doi.org/10. 2151/jmsj.2012-A02

Yukimoto S, Kawai H, Koshiro T, Oshima N, Yoshida K, Urakawa S, Tsujino H, Deushi M, Tanaka T, Hosaka M, Yabu S, Yoshimura H, Shindo E, Mizuta R, Obata A, Adachi Y, Ishii M (2019) The Meteorological Research Institute Earth System Model version 2.0, MRI-ESM2.0: description and basic evaluation of the physical component. J Meteor Soc Japan 97:931-965. https://doi.org/10.2151/jmsj.2019-051

\section{Publisher's Note}

Springer Nature remains neutral with regard to jurisdictional claims in published maps and institutional affiliations.

\section{Submit your manuscript to a SpringerOpen ${ }^{\circ}$ journal and benefit from:}

- Convenient online submission

- Rigorous peer review

- Open access: articles freely available online

- High visibility within the field

- Retaining the copyright to your article

Submit your next manuscript at $\boldsymbol{\nabla}$ springeropen.com 